\title{
THE STRUCTURE OF THE STRONGLY LENSED GAMMA-RAY SOURCE B2 0218+35
}

\author{
Anna Barnacka ${ }^{1,2}$, Margaret J. Geller ${ }^{1}$, Ian P. Dell'Antonio ${ }^{3}$, and Adi Zitrin ${ }^{4}$ \\ ${ }^{1}$ Harvard-Smithsonian Center for Astrophysics, 60 Garden Street, MS-20, Cambridge, MA 02138, USA; abarnacka@cfa.harvard.edu \\ ${ }_{2}$ Astronomical Observatory, Jagiellonian University, Cracow, Poland \\ ${ }^{3}$ Department of Physics, Brown University, Box 1843, Providence, RI 02912, USA \\ ${ }^{4}$ Cahill Center for Astronomy and Astrophysics, California Institute of Technology, MC 249-17, Pasadena, CA 91125, USA \\ Received 2015 November 13; accepted 2016 February 16; published 2016 April 11
}

\begin{abstract}
Strong gravitational lensing is a powerful tool for resolving the high-energy universe. We combine the temporal resolution of Fermi-LAT, the angular resolution of radio telescopes, and the independently and precisely known Hubble constant from the analysis by the Planck collaboration, to resolve the spatial origin of gamma-ray flares in the strongly lensed source B2 $0218+35$. The lensing model achieves 1 mas spatial resolution of the source at gamma-ray energies. The data imply that the gamma-ray flaring sites are separate from the radio core: the bright gamma-ray flare (MJD: 56160-56280) occurred $51 \pm 8$ pc from the $15 \mathrm{GHz}$ radio core, toward the central engine. This displacement is significant at the $\sim 3 \sigma$ level, and is limited primarily by the precision of the Hubble constant. B2 $0218+35$ is the first source where the position of the gamma-ray emitting region relative to the radio core can be resolved. We discuss the potential of an ensemble of strongly lensed high-energy sources for elucidating the physics of distant variable sources based on data from Chandra and SKA.
\end{abstract}

Key words: cosmological parameters - galaxies: jets - gamma rays: general - gravitational lensing: strong quasars: individual (B2 0218+35)

\section{INTRODUCTION}

The high-energy universe is dominated by extreme and violently variable objects. The powerful jets of relativistic plasma associated with these sources are the largest and the most efficient particle accelerators known. The energy source, the energy dissipation mechanism and the particle acceleration mechanism in these fast flares of non-thermal radiation remain puzzling. Proposed mechanisms include relativistic shocks and magnetic reconnection (Zenitani \& Hoshino 2001; Stawarz \& Ostrowski 2002; Kirk \& Skjraasen 2003; Lyutikov \& Uzdensky 2003; Jaroschek et al. 2004; Lyubarsky 2005; Proga 2005; McKinney 2006; Komissarov et al. 2007; Zenitani \& Hoshino 2007; Giannios et al. 2009; Zweibel \& Yamada 2009; Tchekhovskoy et al. 2010, 2011; Nalewajko et al. 2011; Cerutti et al. 2012; Hoshino 2012; Guo et al. 2014, 2015; Sironi \& Spitkovsky 2014; Broderick \& Tchekhovskoy 2015; MacDonald et al. 2015; Moscibrodzka et al. 2015; Nalewajko et al. 2015; Tchekhovskoy \& Bromberg 2015).

One of the limitations to understanding these sources is our inability to localize the spatial origin of the emission. This failure is a direct result of the poor resolution of gamma-ray telescopes that reach, at best, an angular resolution of 0.1 . This angular resolution is unlikely to improve substantially with future instruments because it is limited by fundamental physical effects including nuclear recoil.

Gravitational lensing magnifies distant sources. Thus, lensed gamma-ray blazars offer the best opportunity for resolving the locations of the emitting regions. For example, the bright blazar PKS 1830-211 is a lensed system (Barnacka et al. 2011). Analysis of the time delays between gamma-rays from the mirage images of the flaring episodes, combined with the lens model for this source reveals that two gammaray flares originated from a region within $100 \mathrm{pc}$ from the central engine. Two additional gamma-ray flares originated at least $1.5 \mathrm{kpc}$ from the central engine (Barnacka et al. 2015a). The existence of multiple variable emitting regions along the jet pose challenges for understanding the particle acceleration mechanism.

A second gravitationally lensed blazar B2 $0218+35$ offers further opportunities to explore the origin of the variable gamma-ray emission. This well-observed system has a number of features that enable the derivation of strong constraints on the nature of the gamma-ray source. The lens galaxy, observed with Hubble Space Telescope (HST), is surprisingly simple and isolated (Wucknitz et al. 2004). There are extensive highresolution radio observations of the lensed radio jet at several wavelengths. The Fermi-LAT light curve includes two flares, one of long duration and one short flare.

Based on the optical observations of the lens system and the radio observations of the lensed source, we demonstrate that the positions of the radio core and jet can be localized to 1 mas. Localization of the gamma-ray source relative to the radio emitting regions requires both the time resolution of the FermiLAT light curve and a well-measured Hubble constant (Barnacka et al. 2014a, 2015b).

The Fermi-LAT light curves provide time delays with an accuracy of a few hours. If the time delay originates from the resolved radio core, the associated Hubble constant should be the true value obtained with independent techniques. If there is an offset of even a few miliarcseconds between the gamma-ray emitting region and the radio core, the time delay will imply a Hubble constant that differs from the true value measured with many independent methods (Freedman et al. 2001, 2012; Freedman \& Madore 2010; Suyu et al. 2010, 2013; Riess et al. 2011a, 2011b; Chávez et al. 2012; Efstathiou 2014). Evidently, if the Hubble constant is well-known, the offset between the gamma-ray emitting region and the radio core can be derived with remarkable significance limited only by the accuracy of the time delay and the independently determined Hubble constant (Barnacka et al. 2015b).

We introduce the optical and radio observations of the gravitationally lensed blazar B2 $0218+35$ and we reconstruct the properties of the lens and the source (Sections 2 and 3). In 
Section 4, we use the Fermi-LAT data to explore the gammaray properties of $\mathrm{B} 20218+35$. We focus on two gamma-ray flares where we measure gravitationally induced time delays (Section 5). In Section 6, we combine the measurements of the gamma-ray time delays, the well-resolved position of the radio core, the reconstructed gravitational potential of the lens, and we explore the relative spatial origin of the two gamma-ray flares. Finally, we apply the Hubble parameter tuning (HPT) approach, where we use the independently measured Hubble constant to localize the gamma-ray emission relative to the radio core. We compare the results for $\mathrm{B} 2 \mathrm{0218+35}$ with those for PKS 1830-211. We discuss the implications of the source structure for gamma-ray emission mechanisms in Section 7. We also propose extension of this approach to sources that will be observed with SKA and Chandra. We conclude in Section 8 .

\section{B2 0218+35: A GRAVITATIONALLY LENSED SYSTEM}

B2 $0218+35$ is a gravitationally lensed system with the smallest known Einstein radius (330 mas) (O'Dea et al. 1992; Patnaik et al. 1995). The system consists of a bright blazar at redshift $z_{S}=0.944 \pm 0.002$ (Cohen et al. 2003), lensed by an apparently isolated spiral galaxy at redshift $z=0.6847$ (Browne et al. 1993). The lens bends the emission of the jet into two bright images of the core and extended structures, including an Einstein ring (O'Dea et al. 1992; Patnaik et al. 1992, 1993, 1995; Jackson et al. 2000; Biggs et al. 2001, 2003).

The first measurement of the time delay using VLA $15 \mathrm{GHz}$ polarization observations yielded a value of $12 \pm 3$ days (Corbett et al. 1996). Biggs et al. (1999) used the results of a three-month VLA monitoring campaign at two frequencies and obtained a time delay of $10.5 \pm 0.4$ days. Cohen et al. (2000) used high-precision VLA flux density measurements, over the same epoch as Biggs et al. (1999), and measured a time delay of $10.1_{-1.6}^{+1.5}$ days.

This system has been a "golden lens" for Hubble constant measurement (Wucknitz et al. 2004). However, despite precise measurements of the time delay, a clean lens environment without nearby companions or a surrounding cluster, and a negligible number of structures along the line of sight which would complicate the modeling of the lens, the $H_{0}$ values derived from this system are in the range $61-78 \mathrm{~km} \mathrm{~s}^{-1} \mathrm{Mpc}^{-1}$ (Lehár et al. 2000; Wucknitz et al. 2004; York et al. 2005). The most recent attempt to measure $H_{0}$ for B2 $0218+35$, using a time delay of $11.46 \pm 0.16$ days based on gamma-ray emission, results in a Hubble constant of $64 \pm 4 \mathrm{~km} \mathrm{~s}^{-1} \mathrm{Mpc}^{-1}$ (Cheung et al. 2014).

This large scatter in the $H_{0}$ values can indicate complex source structure (Barnacka et al. 2015b). To investigate the detailed source structure, we build a lens model.

\section{B2 0218+35 AS A HIGH-RESOLUTION COSMIC TELESCOPE}

B2 $0218+35$ is a perfect system for lens modeling. The simplicity and isolation of the lensing galaxy results in a clean gravitational lens potential. Previous lens models show that the observations are consistent with a Singular Isothermal Sphere (SIS) model for the mass distribution of the lens (Wucknitz et al. 2004; Larchenkova et al. 2011). These studies focused on

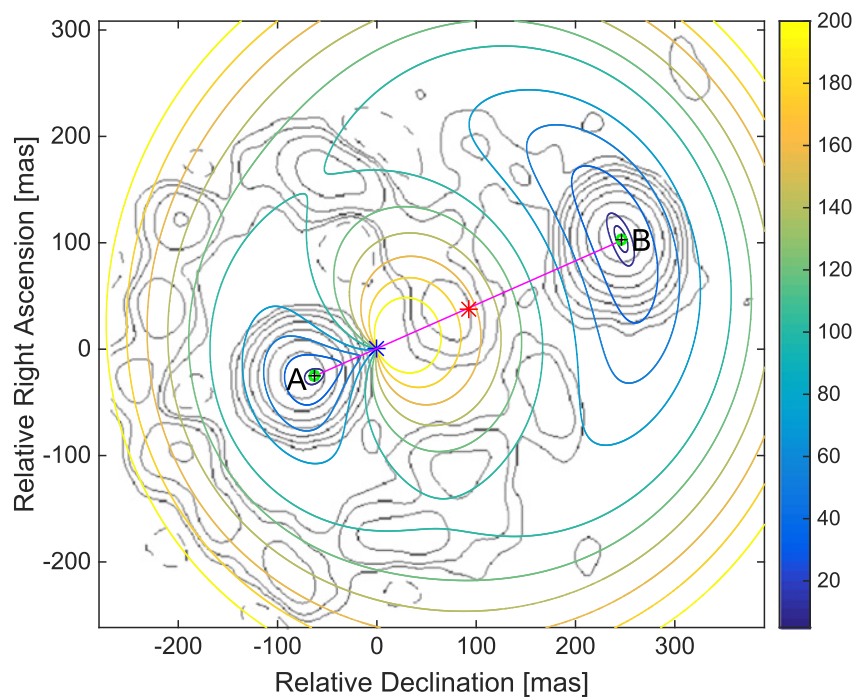

Figure 1. Image plane. The colored contours show the Fermat surface. The coordinates are relative to the reconstructed lens position. Black crosses show the reconstructed mirage image positions. Green open circles show the positions of the $15 \mathrm{GHz}$ mirage images of B2 $0218+35$. The gray contours show the radio emission observed at $1.687 \mathrm{GHz}$. The magenta line indicates the axis connecting the mirage images. The blue star shows the final position of the lens center. The red star indicates the reconstructed position of the $15 \mathrm{GHz}$ radio source.

the properties of the lensing galaxy and measurement of the Hubble parameter (York et al. 2005). Here, we use the lens as a high-resolution telescope to investigate the structure of the source over wavelengths ranging from radio to gamma-ray energies. We first use the lens model to determine an accurate position for the radio core. We evaluate the uncertainties in the model using Monte Carlo simulations.

\subsection{Constraining the Lens}

We base the model on radio VLBA observations at $15 \mathrm{GHz}$, where the position of mirage images of the core are measured with 0.6 mas accuracy (1994 October 3, Patnaik et al. 1995). The position of the mirage image B (brighter image located outside the Einstein ring; green circle in Figure 1) with respect to image A (green circle, Figure 1), along with all the parameters, are summarized in Table 1.

The positions of the $15 \mathrm{GHz}$ mirage images yield a measure of the Einstein radius and the position of the source. The Einstein radius of the lens, with a mass distribution close to a SIS, is half the distance between the mirage images; $\theta_{\mathrm{E}}=\left(\theta_{A}+\theta_{B}\right) / 2=167.2 \pm 0.6$ mas. The corresponding lens mass within one Einstein radius is $\sim 2 \times 10^{10} M_{\odot}$. For a lens of such a small mass, resolution of the mirage images is possible only with high-resolution imaging.

The mirage images appear on the axis defined by the position of the source and the lens (magenta line in Figure 1), at distances of $\pm \theta_{\mathrm{E}}$ from the source. The source is located at half the distance between the mirage images, $\theta_{S}=\left(\theta_{A}-\theta_{B}\right) / 2$. The source position may deviate from the axis if the mass distribution deviates from a SIS. In the model described below, we thus search for the best source position within 10 mas from this estimate.

The last major unknown is the position of the lens. York et al. (2005) derived the optical center of the galaxy with an accuracy of $\sim 15$ mas (see Table 1 ). We seek a center of mass 
Table 1

Input Parameters for the Lens Modeling

\begin{tabular}{lc}
\hline \hline Parameter & Value \\
\hline Image A at $15 \mathrm{GHz}$ & $(0,0)$ \\
Image B at $15 \mathrm{GHz}$ & $(309.2,-127.4) \pm 0.6$ mas $^{\mathrm{a}}$ \\
Estimated source position & $(154.6,-63.7) \pm 10$ mas \\
Einstein angle & $\theta_{\mathrm{E}}=167.2 \pm 0.6$ mas \\
Approximate lens position & $(56,-23) \pm 20$ mas \\
Optical center of the Galaxy & \\
- no masking & $(57 \pm 4,1 \pm 6)$ \\
- spiral arms masked & $(75 \pm 6,-6 \pm 13)$ \\
Position angle & $\phi_{0}=49^{\circ \mathrm{c}}$ \\
Time delay at $15 \mathrm{GHz}$ & $10.1 \pm 1.6$ days $^{\mathrm{d}}$, \\
& $10.5 \pm 0.4$ days $^{\mathrm{e}}$ \\
Magnification ratio at $15 \mathrm{GHz}$ & $3.623 \pm 0.065^{\mathrm{f}}$ \\
Source redshift & $z_{S}=0.944^{\mathrm{g}}$, \\
Lens (Galaxy) redshift & $z_{L}=0.6847^{\mathrm{h}}$ \\
\hline
\end{tabular}

Notes.

${ }^{a}$ Patnaik et al. (1995).

b York et al. (2005).

c York et al. (2005).

${ }^{\mathrm{d}}$ Cohen et al. (2000).

e Biggs et al. (1999).

${ }^{f}$ Patnaik et al. (1995).

g Cohen et al. (2003).

${ }^{\mathrm{h}}$ Browne et al. (1993).

reconstructed to $\sim 1$ mas. We first take the position of the lens as inferred from the optical images. Then, as an additional constraint, we use the lens geometry; the lens must be located close to the image axes and to the center of the Einstein ring (Figure 1).

For demonstration purposes, in Figure 1 we display the contours of radio emission observed at $1.687 \mathrm{GHz}$ with a beam FWHM of $50 \times 50$ mas. However, this image was available to us only in jpg format downloaded from $\mathrm{JVAS}^{5}$. We rescaled it by $\sim 5 \%$ to align it with well-resolved mirage images at $15 \mathrm{GHz}$. The image at $1.687 \mathrm{GHz}$ resolves the mirage images of the core and the Einstein ring structures. After rescaling, the center of the ring indicates a plausible position of the lens with an accuracy of $\sim 50$ mas. Using all of this information, we find the approximate position of the lens (see Table 1). In the lens model described below, we use Monte Carlo simulations to refine the best lens position within this region.

The VLBA observations at $15 \mathrm{GHz}$ also provide a very precise flux density ratio between mirage images of $3.623 \pm 0.065$. However, observations of B2 $0218+35$ at different frequencies and epochs show a large spread in measured magnification ratios, from 1 to 6 (Biggs et al. 1999; Cohen et al. 2000; Cheung et al. 2014). This large spread in magnification ratio may result from different propagation effects (Mittal et al. 2007), substructures in the mass distribution (Mao \& Schneider 1998; Biggs et al. 2004; Kochanek \& Dalal 2004; Metcalf \& Amara 2012), or even from microlensing (Vovk \& Neronov 2015). To avoid this additional complexity, we do not use the flux ratio as a constraint. However, the best-fit model does yield a magnification ratio that is consistent with the range of values observed at other frequencies.

\footnotetext{
5 http://www.jb.man.ac.uk/research/gravlens/lensarch/B0218+357/ $\mathrm{B} 0218+357 . \mathrm{html}$
}

Table 2

Results of the Fit

\begin{tabular}{lc}
\hline \hline Parameter & Value \\
\hline Ellipticity $\epsilon$ & $0.0057 \pm 0.0042$ \\
Source position $\left(x_{S}, y_{S}\right)$ & $(154.2 \pm 0.8,-62.9 \pm 0.7)$ mas \\
Lens position $\left(x_{L}, y_{L}\right)$ & $(62.2 \pm 0.9,-25.0 \pm 0.8)$ mas \\
\hline
\end{tabular}

\subsection{Lens Modeling}

We investigate the properties of the lens system with a MATLAB code inspired by Zitrin et al. (2009, 2013). We construct a $800 \times 800$ mas grid with a resolution of 1 mas. We define coordinates relative to the position of the lens center. We perform all calculations in the image plane because the observed positions of the images are directly linked to the image plane, not to the source plane.

\subsubsection{Gravitational Lensing Formalism}

We compute the Fermat surface using Equation (61) from Narayan \& Bartelmann (1996):

$$
(\boldsymbol{\theta}-\boldsymbol{\beta})-\nabla_{\theta} \psi=0,
$$

where $\boldsymbol{\theta}$ is the position of a mirage image, $\boldsymbol{\beta}$ is the source position, and $\psi$ is the gravitational potential of the lens. The Fermat principle implies that the images form at the extrema (maxima, minima, and saddle points) of the surface (Blandford \& Narayan 1986). We then search for the extrema of the Fermat surface using Mat lab procedure extrema 2. ${ }^{6}$ Figure 2 shows color contours of the Fermat surface with two minima where the mirage images form. We find the positions of these minima and calculate the offset between their coordinates and positions of the mirage images resolved at $15 \mathrm{GHz}$.

The position of the images and the gravitational potential of the lens allow us to calculate the time delay between the mirage images. We use Equation (63) from Narayan \& Bartelmann (1996):

$$
t(\boldsymbol{\theta})=D \frac{\left(1+z_{L}\right)}{c}\left[\frac{1}{2}(\boldsymbol{\theta}-\boldsymbol{\beta})^{2}-\psi(\boldsymbol{\theta})\right],
$$

to calculate the time delay between the arrival of photons from image $\theta_{A}$ and $\theta_{B}$. We then calculate the difference between the estimated time delay and the time delay measured at $15 \mathrm{GHz}$.

We calculate magnifications of the mirage images using Equations (55)-(60) from Blandford \& Narayan (1986). This calculation is also based on the gravitational potential and the positions of the mirage image. We do not, however, use the magnification ratio to extract the lens parameters. We provide the formalism because we do use the magnification later to constrain the origin of the gamma-ray radiation.

\subsubsection{Finding a Unique Mass Model}

We seek the gravitational potential along with source and lens locations that reproduce the observations. We compare the reconstructed positions of the lensed images with well-resolved mirage images at $15 \mathrm{GHz}$. As an additional constrain, we use time delay measured at $15 \mathrm{GHz}$.

\footnotetext{
6 http://www.mathworks.com/matlabcentral/fileexchange/12275-extremam-extrema2-m
} 
To find the lens solution, we repeat our calculations of the image positions and the time delay between them for a range of parameters. We investigated a range of complex models for the gravitational potential using the Monte Carlo simulations. We added parameters including a core, a variable slope for the mass distribution, and a variable ellipticity and position angle of the lensing galaxy. None of the added parameter to the lens model where able to improve the fit and reconstruct the observations with desired accuracy. We vary the lens position around the value listed in Table 1 . We explore a region of 20 mas with a 1 mas step. We search for the best source position around the value listed in Table 1. In the Appendix, we describe our Monte Carlo simulations and our investigation of systematic errors associated with lens model.

We define the best-reconstructed model as the one which reproduces the positions of the mirage images with the smallest offset and where the time delay is within $1 \sigma$ of the measured time delay at $15 \mathrm{GHz}$.

\subsubsection{The Best Model}

We achieved the best reconstruction for an elliptical SIS (Kneib 2014):

$$
\psi(r, \theta)=r \theta_{\mathrm{E}} \sqrt{1-\epsilon \cos \left(2\left(\phi-\phi_{0}\right)\right)},
$$

where $\epsilon$ is an ellipticity of the gravitational potential, $\phi_{0}$ is the position angle of the potential, and $\theta_{\mathrm{E}}$ is an Einstein angle defined as

$$
\theta_{\mathrm{E}}=4 \pi \frac{\sigma_{0}^{2}}{c^{2}} \frac{D_{\mathrm{LS}}}{D_{\mathrm{OS}}},
$$

where $\sigma_{0}$ is the central velocity dispersion of the 3D velocity field, and $D_{\mathrm{LS}}$ and $D_{\mathrm{OS}}$ are cosmological distances from the lens to the source, and from the observer to the source, respectively. We also define

$$
D \equiv \frac{D_{\mathrm{OL}} D_{\mathrm{OS}}}{D_{\mathrm{LS}}}=h d,
$$

where $D_{\mathrm{OL}}$ is the distance from the observer to the lens. The parameter $h$ refers to the Hubble constant, $H_{0}=$ $h \times 100 \mathrm{~km} \mathrm{~s}^{-1} \mathrm{Mpc}^{-1}$. We calculate distances based on a homogenous Friedmann-Lemaître-Robertson-Walker cosmology, using $h=0.673$, the mean mass density $\Omega_{M}=0.315$ and the normalized cosmological constant $\Omega_{\Lambda}=0.686$ (Planck Collaboration et al. 2014).

\subsubsection{Error Estimation}

To estimate statistical errors, we use Monte Carlo chain simulations. We based our algorithm on the MCMC toolbox for Matlab (Haario et al. 2006).

We test for systematics in our simulations by comparing the numerical solution with an analytic model. The simplest analytic solution is the SIS. We compare the positions of the images, time delays and magnification ratios for the SIS calculated analytically and numerically for positions of the sources across the entire lens plane. The numerical procedure applied on a grid with a 1 mas resolution, on average reconstructs image positions with $\sim 0.3$ mas. On average, the time delay is reproduced within 0.01 days, and the

\footnotetext{
7 http://helios.fmi.fi/ lainema/mcmc/
}

Table 3

Reconstruction

\begin{tabular}{lcc}
\hline \hline Parameter & Value & Difference \\
\hline Image A & $(-0.4,0)$ & 0.4 mas \\
Image B & $(308.6,-128.0)$ & 0.85 mas \\
Time delay & 10.7 days & $\sim 0.2$ days \\
Magnification ratio & 3.85 & 0.23 \\
\hline
\end{tabular}

magnification ratio within 0.05 . This level of precision shows that on a scale of 1 mas, our lens parameters are unaffected by systematic numerical errors.

\subsubsection{Lens Modeling Results}

Table 1 summarizes the input parameters for the lens model; the ellipticity of the lens, and the source and lens positions. Table 2 shows the model results along with the statistical errors from the Monte Carlo chain simulations.

The fit yields an $\epsilon \sim 0$, essentially an isotropic SIS. We reconstruct the lens and source positions with an accuracy of 1 mas corresponding to $8 \mathrm{pc}$ in the source plane.

The positions of the mirage images are most sensitive to changes of the source position in the tangential direction relative to the images-lens axis. Changing the source position by 1 mas in the tangential direction, moves the mirage images by 5.5 mas. In the radial direction a 1 mas change in the source position displaces the image by only 2.7 mas. Table 3 and Figure 1 show that the model reproduces the observed mirage image positions to $0.4-0.8$ mas.

\subsection{Lens Model and the Jet Alignment}

We investigate the origin of variable emission along the relativistic jet. The alignment of the jet and the mass distribution of the lens are necessary to predict the range of time delays and corresponding magnification ratios.

The alignment of the jet of B2 $0218+35$ is known from the well-resolved radio images which show clear jet-like structures. Wucknitz et al. (2004) show that the jet sub-components are oriented exactly radially with respect to the center of mass of the lens. The existence of the radio Einstein ring implies radial alignment of the jet on scales $\lesssim \mathrm{kpc}$.

We use the alignment of the jet and the lens model (Table 2) to calculate the time delay and corresponding magnification ratio along the jet. Figure 2 shows the result.

The time delay is very sensitive to the distance of the source from the center of the lens. The radial alignment of the jet of B2 $0218+35$ produces maximal differences in the time delay among regions distributed along the jet. In the radial direction the time delay changes by 0.13 days $(3.12 \mathrm{hr})$ per 1 mas but in the tangential direction, it changes much more slowly, 0.01 day per 1 mas.

\section{B2 0218+35 AS A GAMMA-RAY SOURCE}

B2 $0218+35$ is a bright gamma-ray source. Here, we analyze the gamma-ray light curve observed with the Large Area Telescope onboard the Fermi mission (Fermi-LAT, Atwood et al. 2009).

We analyze the Fermi-LAT P7REP events and spacecraft data of B2 $0218+35$ during the period MJD: 54682-57041. We use the standard likelihood tools distributed with the 

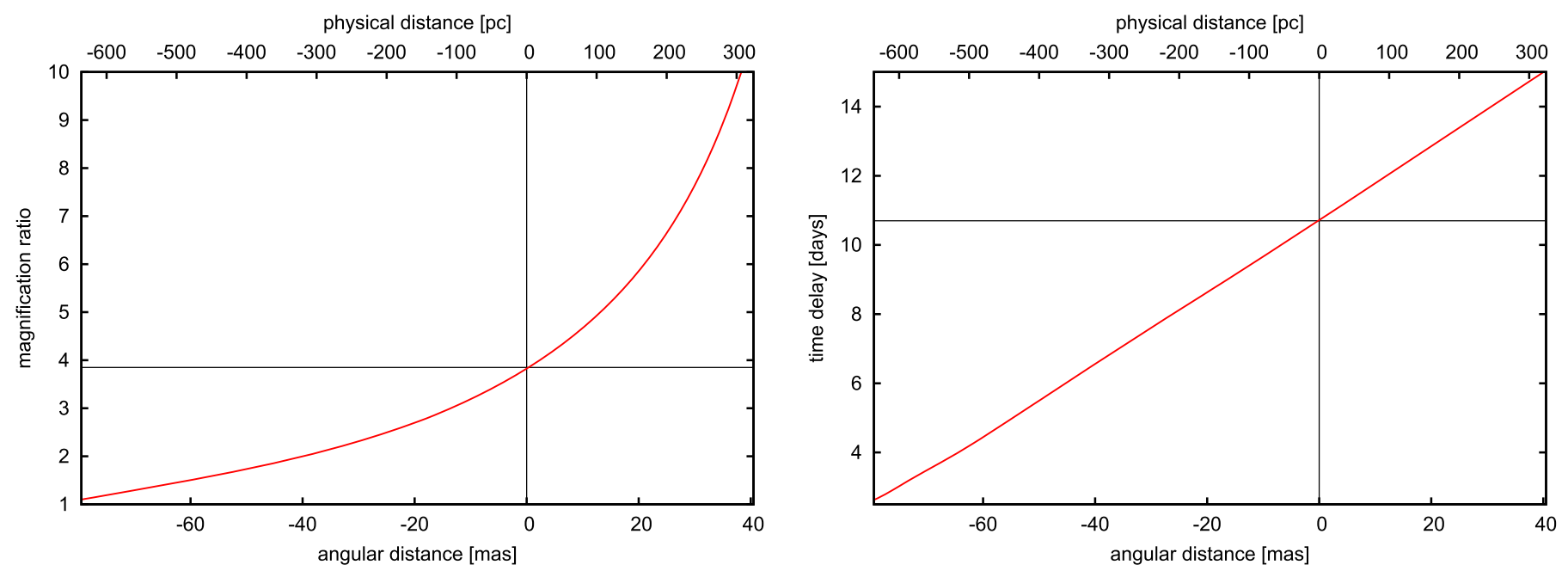

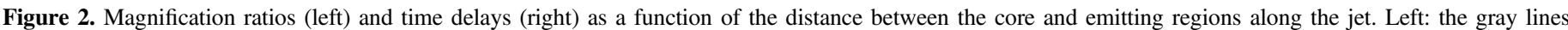

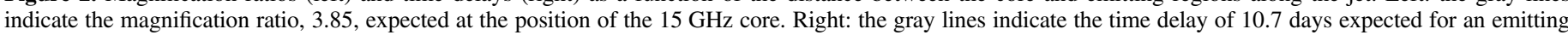
region coincident with the position of the $15 \mathrm{GHz}$ core.

Science Tools v9r32p5 package available on the Fermi Science Support Center webpage.

We only used events in the CLEAN data set with the highest probability of being photons. We exclude events with zenith angles $>100^{\circ}$ to limit contamination by Earth albedo gammarays produced by cosmic rays interacting with the upper atmosphere. We also remove events with rocking angles $>52^{\circ}$ to eliminate time intervals when the Earth entered the LAT Field of View.

The selected events with reconstructed energies above $100 \mathrm{MeV}$ within a square region of $10^{\circ}$ radius are centered on the coordinates of B2 $0218+35$ (see Figure 3). We analyze the selected photons with a binned maximum likelihood method (Mattox et al. 1996).

We model the background emission using a galactic diffuse emission model ( $\left.g l I_{-} i e m \_v 05\right)$ with an isotropic component (iso_clean_v05; available on the Fermi Science Support Center webpage). The fluxes are based on the post-launch instrument response function P7REP_CLEAN_V15.

The XML source model contains all of the sources included in the Second Fermi/LAT catalog (Nolan et al. 2012) within a radius of $20^{\circ}$ around $\mathrm{B} 20218+35$. We first analyze the XML source model fitting the sources within $10^{\circ}$; within an annulus from $10^{\circ}$ to $20^{\circ}$ we fix the sources to their $2 \mathrm{FGL}$ values. We calculate the test statistic (TS) for all the sources located within the $10^{\circ}$ radius during the time period MJD: 54682-57041. Sources with a TS lower than 6.5 , corresponding to a statistical significance of $\approx 2.5 \sigma$, are then fixed in further analysis. The resulting XML source model is then the basis for the 6.5 year light curve in Figure 4. The TS is also based on this source model.

The TS of B2 $0218+35$ for this data set is 8890 , corresponding to a statistical significance of $\approx 94 \sigma$. The energy spectrum is best described by a power law with $\Gamma=2.28 \pm 0.02$ and an integral flux of $F(0.1-300 \mathrm{GeV})=(1.42 \pm 0.05) \times$ $10^{-7} \mathrm{ph} \mathrm{cm}^{-2} \mathrm{~s}^{-1}$. The highest energy event recorded by Fermi/LAT corresponds to $\sim 95.7 \mathrm{GeV}$. The flux in each time bin is reconstructed in the same manner as for the full time range; the photon index and integral flux in these bands are free parameters.

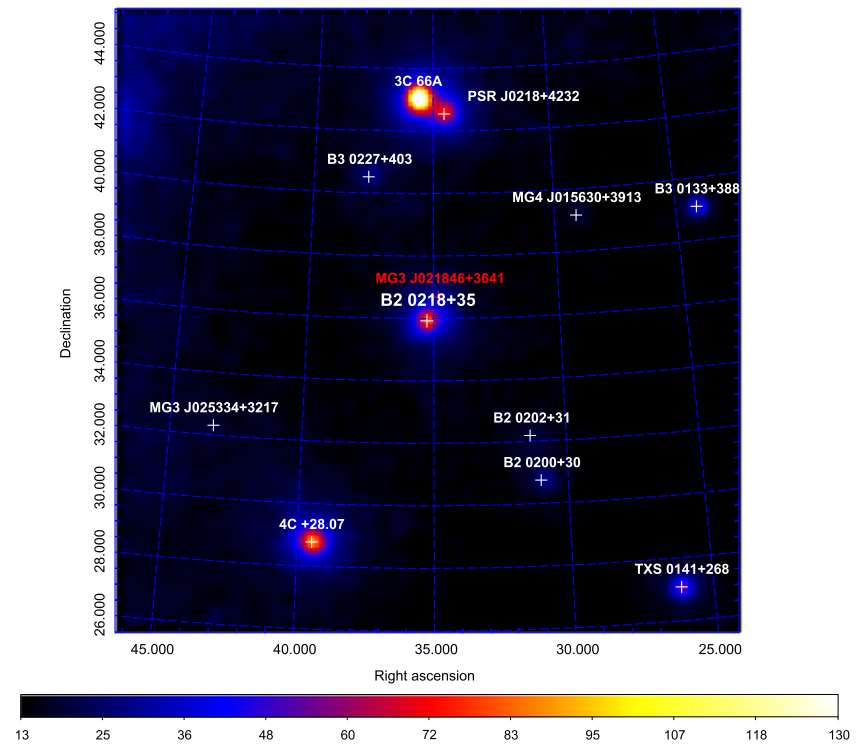

Figure 3. Fermi-LAT count map of $\mathrm{B} 20218+35$. The energy range is $100 \mathrm{MeV}-300 \mathrm{GeV}$.

Our goal is to investigate the spatial origin of gamma-ray flares. Thus, we look for periods of flaring activity in the gamma-ray light curve of B2 $0218+35$. We define a flare as a period of time when the emission in a one-day bin increases by at least two sigma relative to the average flux. Note that Figure 4 shows data in seven day bins.

Based on our definition, we identify two active periods: a very long flare between MJD: 56160-56280, and a short flare consisting of a single bright event, occurring between MJD: 56800-57000. Figure 5 shows both flares. Visually it may seem that there are other flares in Figure 4. However, these apparent flares are not significant with one-day bins. We use the light curves for the two significant flares to compute time delays.

\section{TIME DELAY MEASUREMENT}

In the B2 $0218+35$ system, radio observations reveal two images separated by $\sim 330$ mas. The angular resolution of the 


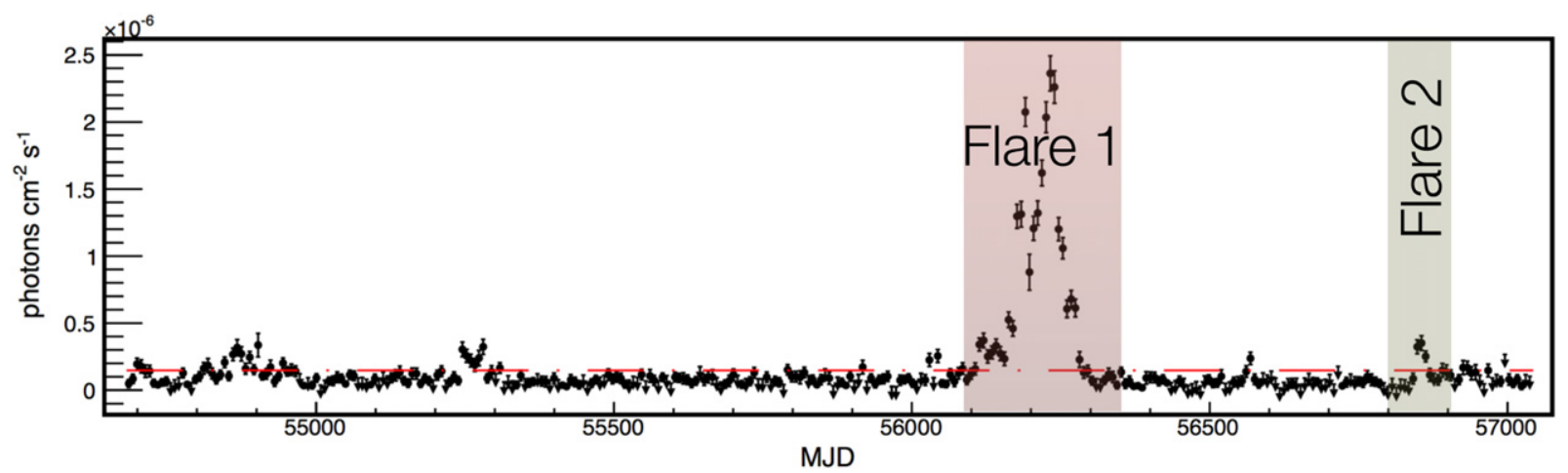

Figure 4. Fermi-LAT light curve of B2 $0218+35$ with seven-day binning. The energy range is $100 \mathrm{MeV}-300 \mathrm{GeV}$.
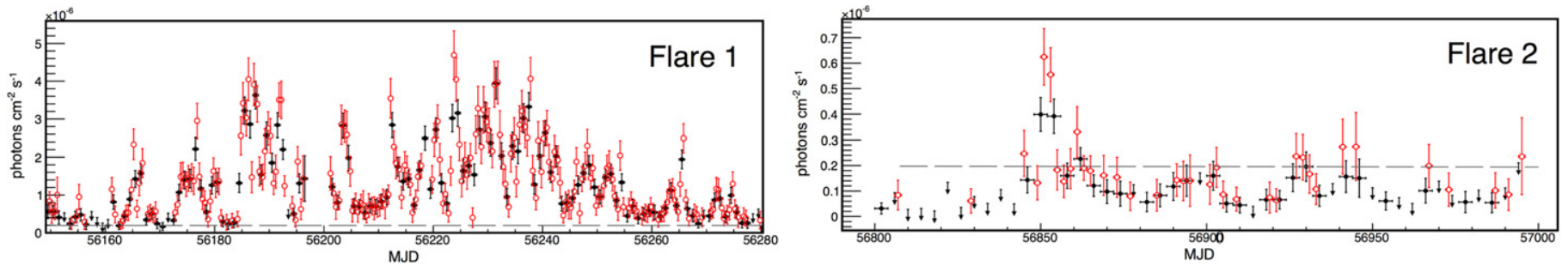

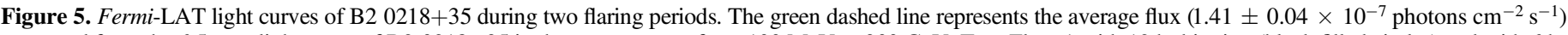

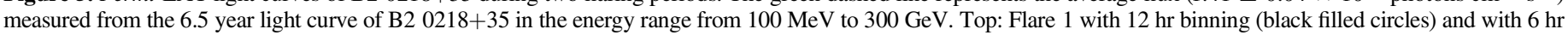
binning (red open circles). Bottom: Flare 2 with four-day binning (black filled circles), and two-day binning (red open circles).

Fermi-LAT detector is $\sim 1^{\circ}$. Thus the observed gamma-ray light curve is a sum of two unresolved mirage images shifted in time, but with a constant magnification ratio. The temporal resolution of the Fermi-LAT detector allows determination of the time delay at gamma-ray energies. With sufficiently welldetermined time delays, the relationship between the radio and gamma-ray time delays constrain the relative source positions.

The Fermi-LAT data cover a long, uninterrupted periods containing the two significant flares. There are several methods for extracting the time delay from these binned data (Barnacka et al. 2015a). We use the standard autocorrelation function (ACF), and the more sensitive double power spectrum (DPS) method (Barnacka et al. 2011; Barnacka 2013). The detailed description of the DPS method together with comparison to other methods is described in Barnacka et al. (2015a, Section 3.2.2 and Appendix). The DPS is similar to the Cepstrum method (Bogert et al. 1963), where a time series with a delay transforms into Fourier space with the extra component $e^{-2 \pi i f a}$, where $f$ is frequency and $a$ is the delay. Squaring the absolute value of this extra component results in a periodic pattern imprinted on the power spectrum. The period of this pattern in the frequency domain is the inverse of the relative time delay $a$. We identify the time delay by calculating and analyzing the power spectrum of the power spectrum that includes the periodic pattern. We apply these methods to the first flaring period in Section 5.1.

Monte Carlo simulations demonstrate that this signal processing allows removal of the intrinsic variability of the source. We have previously demonstrated that this procedure yields precise and significant estimates of the time delays (Barnacka et al. 2015a).

The DPS method is very efficient when applied to long, evenly sampled light curves. However, sometimes the flare is an isolated event of very short duration like Flare 2. To treat these flares, we use the Maximum Peak Method (MPM, Section 3.2.3, Barnacka et al. 2015a) where we calculate the ratio between the flux in the flare and flux in the subsequent data. We compare the flux ratios as a function of the lag between the brightest flare and the subsequent light curve with the predictions of the model (Section 5.2) of B2 $0218+35$.

\subsection{Flare 1}

Enormous gamma-ray activity occurred during the period MJD: 56160-56280. We analyze this time interval using the ACF and DPS (see Figure 6). We obtain a time delay of $11.5 \pm 0.5$ days for the ACF, and $11.38 \pm 0.13$ days using the DPS.

To estimate the significance of the detection, we use Monte Carlo simulations following Barnacka et al. (2015a). We produce $10^{6}$ artificial light curves.

The temporal behavior of Flare 1 is not power-law noise. The flare consists of a superposition of very short duration flares with large amplitudes. Figure 7 compares Flare 1 with the temporal behavior of power-law noise with different indices. The temporal behavior during Flare 1 is not well reproduced by any of these pure noise models. Pink noise $(\alpha \sim 1)$ is the closest match. The power spectrum of Flare 1 actually returns $\alpha=0.9$. However, pink noise is not complete description of temporal behavior of Flare 1 because it cannot account for correlated bin-to-bin time variations of large amplitude. Increasing the index of power-law noise (e.g., red noise) smooths the large fluctuations and obviously does not reproduce the behavior of the source. Decreasing the index toward white noise increases the fluctuations on bin-to-bin time scales, but the fast rise exponential decay profile that characterizes flares is absent (Peng et al. 2010; Saito et al. 2013). This correlated behavior resulting from the 

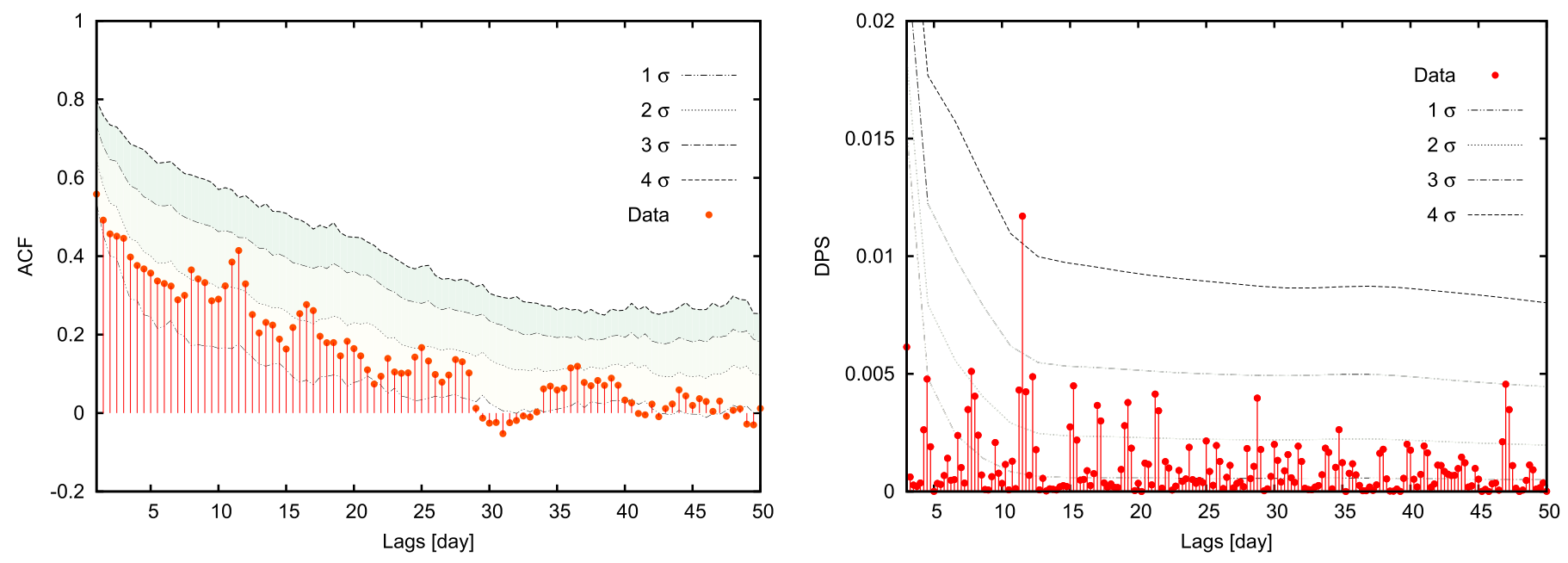

Figure 6. ACF (left) and DPS (right) for Flare 1.

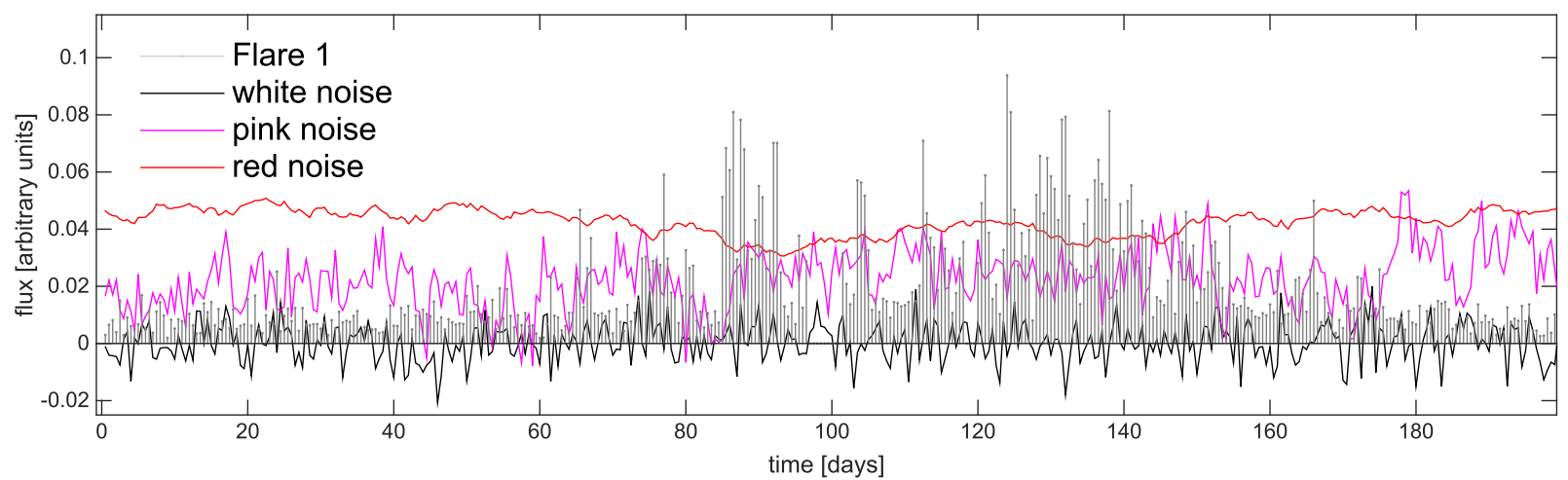

Figure 7. Comparison of the temporal behavior of Flare 1 and power-law noise: white noise $(\alpha \sim 0)$, pink noise $(\alpha \sim 1)$, and red noise $(\alpha \sim 2)$. We scale the flux of the Flare 1 by a factor of $2 \times 10^{4}$ to align the data with the simulations. The flux of the pink and red noise are shifted by a factor of 0.02 and 0.04 , respectively. The shift in scale avoids overlapping of the plots and facilitates comparison of the temporal structure.
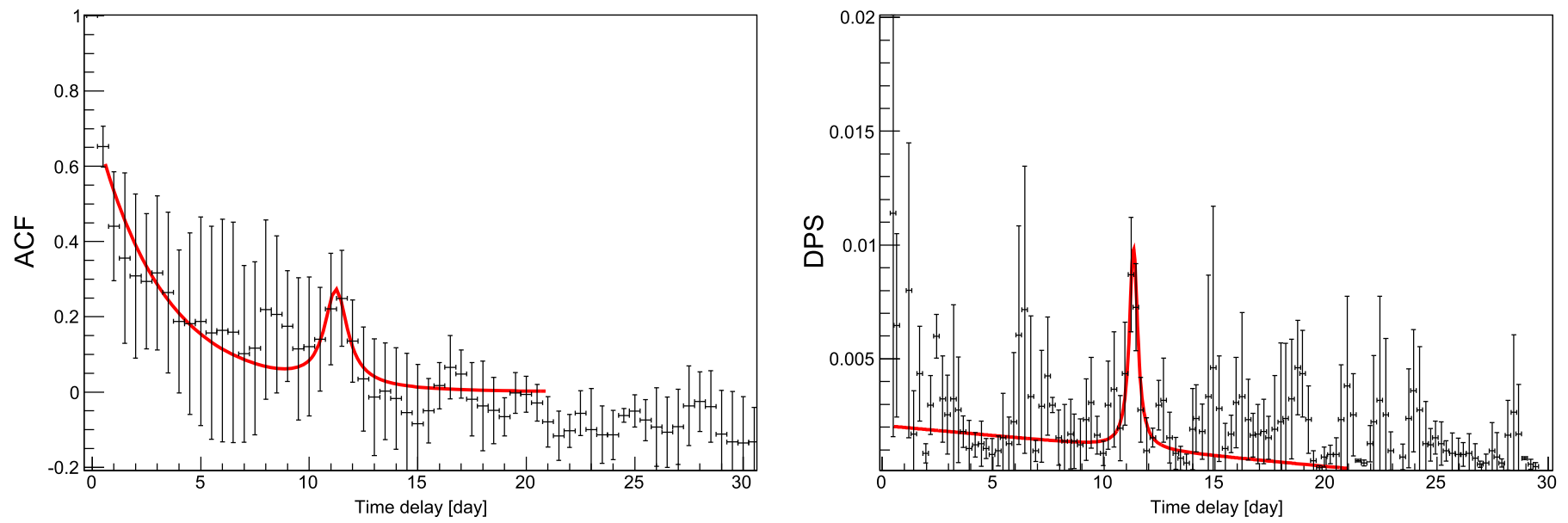

Figure 8. ACF (left) and DPS (right) for Flare 1. The spectra are the average of three time sequences. Solid lines are model fits, as described in the text.

physics of the source is not reproduced by a simple noise model.

Simulations of the signal composed of superpositions of short and bright flares are possible, but the number of parameters required to define the flares is large. Thus following Barnacka et al. (2011), we divide the light curve into overlapping segments. We can use this approach because the flaring activity lasted for almost 200 days and the range of expected time delays is short, $<20$ days. We can thus divide the light curve of Flare 1 into three overlapping segments of 128 days each. We apply the ACF and DPS to each of the three segments and then average the results. The error bars are the standard deviation among the three segments in each bin. 


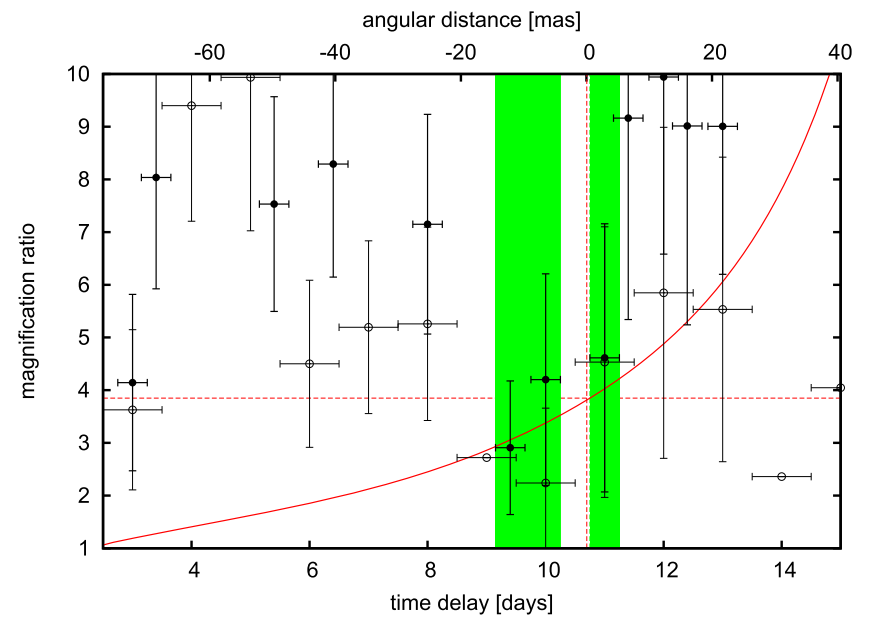

Figure 9. Maximum Peak Method applied to Flare 2. Black and gray points are flux ratios calculated relative to the time bin center at MJD: 56852, using light curve with 0.5 day and 1 day binning, respectively. The solid red line indicates the predicted magnification ratio as a function of the time delay along the jet axis. The red dotted lines indicates the value of the magnification ratio and time delay corresponding to the radio core resolved at $15 \mathrm{GHz}$. The green area indicates values of time delay where the observed flux ratio is consisted with the model.

Figure 8 (Left) shows the ACF averaged over the segments. We fit the spectrum with an exponential function representing the background and a Lorentzian function representing the signal. The fit returns a time delay of $11.24 \pm 0.39$ days. However, the error bars are large and the significance of the signal is only $0.3 \sigma$.

Figure 8 (Right) shows the DPS from the averaged segments. We fit linear plus Lorentzian profiles to obtain the position of the peak and its significance. The corresponding time delay is $11.33 \pm 0.12$ days. The signal is $4.13 \sigma$ above the background. The DPS time delay, detected at high significance, agrees remarkably well with the previously obtained gamma-ray time delay of $11.46 \pm 0.16$ days reported by Cheung et al. (2014).

The light curve is a superposition of multiple flares. The time between the random flares could mimic a time delay. These "fake" signals should occur only in a fraction of the light curve. A true gravitationally induced time delay persists over the entire flaring period. The analysis that averages over segments of the light curve distinguishes the real, gravitationally induced, signal from randomly superimposed flares. If there is a real time delay the significance of the time delay increases with averaging over the three periods. For random multiple flares, the significance should not improve. In fact Figure 8 shows that multiple peaks are present because there is a lot of structure in the light curve. However, only the signal in bins around 11.5 days is significant (Figure 8).

\subsection{Flare 2}

Flare 2, a single, bright flare, occurred in time period MJD: 56800-57000. The light curve (2 day bins) around the flare consists mostly of upper limits (Figure 5). This light curve is useless for extracting several-day long time delays. However, the huge advantage of having a single isolated flare is the ease of a direct search for the echo flare. Figure 9 shows the result of application of the MPM, (Section 3.2.3, Barnacka et al. 2015a). The MPM method suggests that the time delay lies in one of two ranges: $9.75 \pm 0.5$ days or $11 \pm 0.25$ days. The errors corresponds to the bin width, not the $1 \sigma$ standard deviation.

\section{THE STRUCTURE OF THE GAMMA-RAY SOURCE}

So far we have used the radio observations and a lens model to reconstruct the origin of the radio core with a resolution of 1 mas (Section 3.2.5). The Fermi-LAT observations enable precise determination of the time delay for two gamma-ray flares (Section 5). Here, we locate the sources of gamma-ray emission relative to the radio core by combining the radio source map and the Fermi-LAT time delays with the wellmeasured Hubble constant from Planck Collaboration et al. (2014).

Barnacka et al. (2015b) show that the Hubble parameter implied by the time delay is sensitive to any spatial offset between the emission region that produces the resolved mirage images and the site of the variable emission used to measure time delays. Purely on the basis of the physical processes involved, the gamma-ray emission from B2 $0218+35$ may not be spatially coincident with the radio core (Barnacka et al. 2014a).

The Hubble parameter, well measured with a variety of independent methods, provides a route to exploring this issue. We can use this precisely measured Hubble parameter to evaluate any offset between the radio core and the site of the variable gamma-ray emission. We call this method the HPT approach.

The Hubble parameter enters into the distance ratio in the time delay calculation (Equation (2)). For an SIS gravitational potential, the relation reduces to

$$
h=\frac{d\left(1+z_{L}\right)\left(\theta_{B}^{2}-\theta_{A}^{2}\right)}{2 c \Delta t} .
$$

We have three kinds of constraints on the map of the source from radio to gamma-ray wavelengths: the Hubble parameter, the positions of the lensed images, and the time delay between the images $\Delta t$. If there is an offset between the radio core and the gamma-ray emitting regions, the Hubble parameter derived from the Fermi-LAT time delay will differ from the independently measured "true" value. This difference depends on the distance between the radio core and the spatial location of the flare. The offset in Hubble space corresponds to the spatial offset in the source plane (Barnacka et al. 2015b).

To locate the origin of the gamma-ray flares from B2 0218 +35 , we first fix $\theta_{A}$ and $\theta_{B}$ to the positions of the resolved images of the $15 \mathrm{GHz}$ radio core (Table 1). We use these image positions along with the model of the lens and the cosmological parameters to infer the expected time delay for the position of the $15 \mathrm{GHz}$ radio core. Table 3 lists the reconstructed position; the value agrees well with the time delay derived from the variability of the radio core although we do not use this delay to compute the Hubble constant. The reconstructed time delay (Table 3) plugged into Equation (6) is a consistency check which returns the true value of the Hubble parameter, our reference point.

Next, we calculate time delays for positions within $\sim 10$ mas from the radio core. We use these time delays and positions of the $15 \mathrm{GHz}$ images to compute the Hubble parameter using Equation (6). Figure 10 shows these calculated Hubble parameters as a function of the position of the variable emitting region. We call this projection of the model Hubble space. 


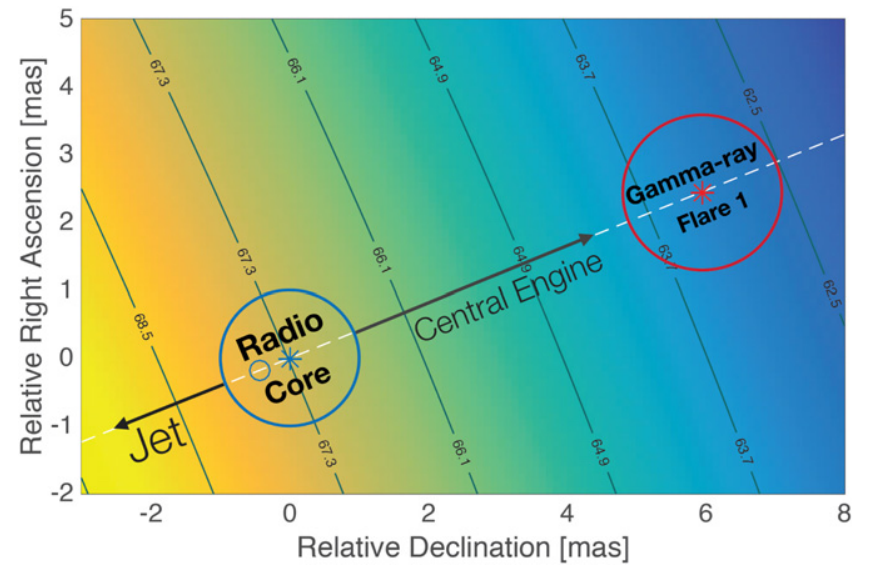

Figure 10. Hubble space. The distances are shown with respect to the position of the radio core (blue circle). The radius of the blue circle corresponds to an uncertainty of 1 mas. The blue star indicates the value of the Hubble parameter based on the reconstructed position of the $15 \mathrm{GHz}$ radio core. The open blue point shows the Hubble parameter derived from the observed positions of the $15 \mathrm{GHz}$ radio images. The dotted line shows the jet projection. Gray arrows show the direction from the radio core toward the central engine and toward the jet. The red circle locates the spatial origin of Flare 1 . The radius of the red circle corresponds to the uncertainty in the time delay. The spacing of the white lines in Hubble space corresponds to $1.2 \mathrm{~km} \mathrm{~s}^{-1} \mathrm{Mpc}^{-1}$, the $1 \sigma$ uncertainty in the Hubble parameter (Planck Collaboration et al. 2014).

\subsection{The Spatial Origin of Flare 1}

Flare 1 has a time delay of $11.33 \pm 0.12$ days. The Hubble parameter obtained based on the position of the $15 \mathrm{GHz}$ radio core and this time delay corresponds to $H_{0}=63.64 \pm 0.67 \mathrm{~km} \mathrm{~s}^{-1}$ $\mathrm{Mpc}^{-1}$. The quoted error corresponds to an error in a time delay of 0.12 days that translates into a spatial resolution of 1.15 mas. Recall that $H_{0}=67.3 \pm 1.2 \mathrm{~km} \mathrm{~s}^{-1} \mathrm{Mpc}^{-1}$ from Planck Collaboration et al. (2014).

We indicate the position of the $15 \mathrm{GHz}$ radio core in Hubble space in Figure 10. The Hubble parameter estimated for Flare 1 appears as a red dot in Figure 10. The position of Flare 1 in Hubble space is displaced from the radio core. The resolved radio images also constrain the alignment of the jet as indicated in Figure 10 (white dotted line).

The distance between the $15 \mathrm{GHz}$ core and the site of the gamma-ray flare is $6.4 \pm 1.1$ mas displaced toward the central engine. This displacement corresponds to a projected distance of $51.2 \pm 8.8 \mathrm{pc}$. The accuracy of the Hubble parameter measured with Planck Collaboration et al. (2014), $\pm 1.2 \mathrm{~km} \mathrm{~s}^{-1}$ $\mathrm{Mpc}^{-1}$, implies that the offset between the resolved radio core and the variable gamma-ray site is significant at the $\sim 3 \sigma$ level.

\subsection{The Spatial Origin of Flare 2}

Flare 2 has a time delay in one of two ranges: 10.75-11.25 days or 9.25-10.25 days. For the first range, the Hubble parameter is $63.64-66.6 \mathrm{~km} \mathrm{~s}^{-1} \mathrm{Mpc}^{-1}$. The second range results in a Hubble parameter of $69.85-77.4 \mathrm{~km} \mathrm{~s}^{-1} \mathrm{Mpc}^{-1}$. We indicate the possible sites of Flare 2 in Figure 11. Flare 2 originates either $3.35 \pm 2.30$ mas $(26.8 \pm 18.4 \mathrm{pc}$ in the source plane) from the core toward the central engine, or $8.33 \pm 4.5$ mas $(66.64 \pm 36.00 \mathrm{pc}$ in the source plane $)$ in the direction of the jet.

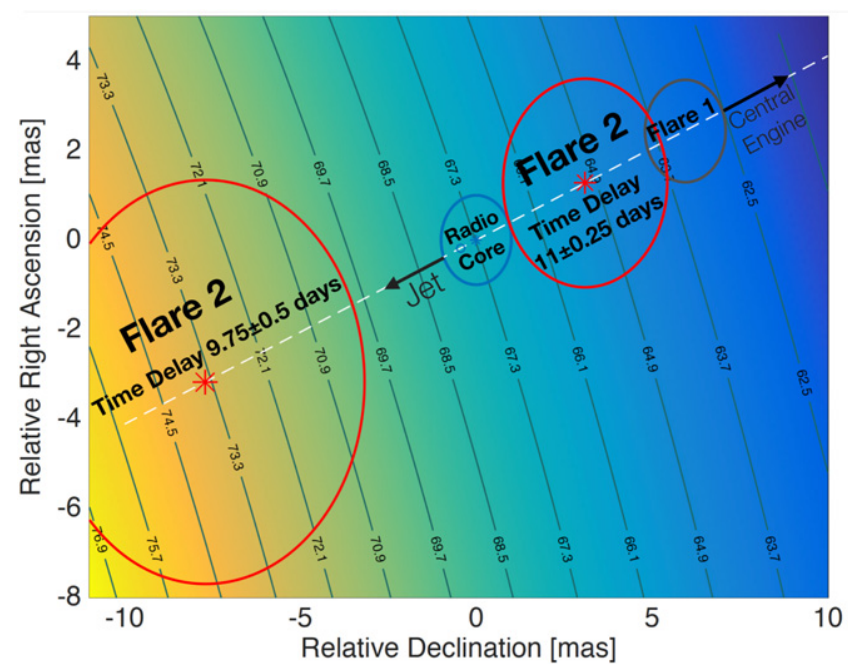

Figure 11. Hubble space calculated as in Figure 10. Red circles show the two possible sites for the spatial origin of Flare 2 corresponding to a time delay of $9.75 \pm 0.5$ days or $11 \pm 0.25$ days.

\subsection{The Connection Between Flare 1 and Flare 2}

The position in the space of Hubble parameter versus offset shows that Flare 2 is not coincident with either the core or Flare 1. Using the HPT approach, we can ask whether Flare 2 could result from a moving knot, which first produced Flare 1 and then moved downstream along the jet to produce Flare 2.

The time between the beginning of Flare 1 and Flare 2, $\Delta t_{\mathrm{obs}}$, is 690 days. The projected distance between Flare 1 and Flare 2, constrained by the time delay of $11 \pm 0.25$ days, is $D_{\text {projected }} \sim 24$ pc. In this case the model implies that knot is moving relativistically with an apparent velocity of $\beta_{\mathrm{app}}$ :

$$
\begin{aligned}
\beta_{\text {app }} & =\frac{D_{\text {projected }}\left(1+z_{S}\right)}{c \Delta t_{\mathrm{obs}}} \\
& \approx 70\left(\frac{D_{\text {projected }}}{24 \mathrm{pc}}\right)\left(\frac{\Delta t_{\mathrm{obs}}}{690 \text { days }}\right) .
\end{aligned}
$$

Similar superluminal apparent motions of $\sim 46 c$ occur, for example, in the radio jet of the blazar PKS 1510-089 (Jorstad et al. 2005). Very high superluminal apparent motions are commonly observed in gamma-ray blazar (Lister et al. 2013, 2015). This time delay thus yields a reasonable physical model for the gamma-ray source.

If the plasmon continues its motion with the same apparent velocity, 1.6 mas $\mathrm{yr}^{-1}$, it will pass through the stationary shock of the $15 \mathrm{GHz}$ core $\sim 2 \pm 1$ years after Flare 2, which was detected in 2014 July. This model thus predicts increased radio emission in the time period around 2016 July. Radio observations during this period could thus provide valuable insight into the physical processes and plasma propagation along the jet.

The second possible site of Flare 2, implied by the time delay of $\sim 9.75 \pm 0.5$, is located at a projected distance of $\sim 16$ mas from Flare 1 . An apparent velocity of $350 c$ would be required to explain such a large projected distance. Thus, these flares could not be produced by the same moving knot of plasma.

We do not have direct evidence that Flare 2 is indeed connected with Flare 1. However, the longer time delay implies 
Table 4

Range of Parameters for the Monte Carlo Simulations

\begin{tabular}{lcc}
\hline \hline Parameter & Min & Max \\
\hline$s$ [mas] & 0 & 50 \\
$q$ & 0.9 & 1 \\
$\alpha$ & 0.96 & 1.04 \\
$x_{S}$ & 350 & 370 \\
$y_{S}$ & 332.5 & 352.5 \\
$x_{L}$ & 248 & 288 \\
$y_{L}$ & 284.6 & 324.6 \\
\hline
\end{tabular}

a reasonable physical model for the source and demonstrates the power of the HPT approach.

\section{DISCUSSION}

A major challenge of gamma-ray astronomy is localization of the emission region. The blazar B2 $0218+35$ is uniquely suited to detailed reconstruction of the source position. The gravitational lensing system is remarkably simple. There are exquisite radio data at several wavelengths along with the extensive Fermi-LAT light curve. These observations combined with the well-constrained Hubble constant enable the first reconstruction of the gamma-ray source positions relative to the radio core and jet.

There are plausible sources of systematics that could in principle account for the the offset between the radio core and gamma-ray emission in B2 $0218+35$. First, we consider a more complex mass distribution for the lens. However, the time delay measured for the resolved radio core $(10.5 \pm 0.4$ days, Biggs et al. 1999) differs from the time delay measured for gamma-ray flares (11.33 \pm 0.16 days). This difference clearly indicates that the complexity is in the source, not the lens. Even complex lens models cannot account for multiple time delays. Moreover, we have investigated a range of complex lens model. More complex lens models were unable to reproduce the observations as well as the SIS model.

Although the time delay difference rules out complexity of the lens, the accuracy of radio time delay ( 0.4 days) allows us to separate the radio core from the gamma-ray flare site only at the $\sim 2 \sigma$ level. The time delay can be translated into a relative position in the source plane. The accuracy of 0.4 days corresponds to $\sim 3$ mas. We use the position of the radio images reconstructed with 1 mas resolution to measure the distance between the emission sites $(51.2 \pm 8.8 \mathrm{pc})$, and we obtain a separation significant at the $\sim 3 \sigma$ level.

We next consider systematics of the Hubble constant measurement. To measure the offset we use a Hubble parameter, $H_{0}=67.3 \pm 1.2 \mathrm{~km} \mathrm{~s}^{-1} \mathrm{Mpc}^{-1}$, obtained by Planck Collaboration et al. (2014). Many independent methods provide a measure of $H_{0}$. For example the $H S T$ Key Project provides $H_{0}=72 \pm 8 \mathrm{~km} \mathrm{~s}^{-1} \mathrm{Mpc}^{-1}$ (Freedman et al. 2001), the Cepheid distance ladder gives $73.8 \pm 2.4 \mathrm{~km} \mathrm{~s}^{-1} \mathrm{Mpc}^{-1}$ (Riess et al. 2011a, 2011b) and 74.3 \pm 1.5 (stat) \pm 2.1(sys) $\mathrm{km} \mathrm{s}^{-1} \mathrm{Mpc}^{-1}$ (Freedman et al. 2012).

The gamma-ray time delay combined with the position of the radio core gives a Hubble parameter of $H_{0}=$ $63.64 \pm 0.67 \mathrm{~km} \mathrm{~s}^{-1} \mathrm{Mpc}^{-1}$. Thus, even if we use the largest value $\left(74.3 \pm 1.5\right.$ (stat) \pm 2.1 (sys) $\mathrm{km} \mathrm{s}^{-1} \mathrm{Mpc}^{-1}$ ) we obtain a significant offset between the radio core and the gamma-ray of at least $\sim 3 \sigma$. Therefore, the separation between the radio core
Table 5

Results of the Monte Carlo Simulations

\begin{tabular}{cccccr}
\hline \hline Parameter & Mean $^{\mathrm{a}}$ & std $^{\mathrm{b}}$ & MCerr $^{\mathrm{c}}$ & $\tau^{\mathrm{d}}$ & geweke $^{\mathrm{e}}$ \\
\hline$s$ & 6.5639 & 4.5181 & 0.1014 & 28.90 & 0.971 \\
$q$ & 0.9925 & 0.0055 & 0.0001 & 25.19 & 0.999 \\
$\alpha$ & 1.0005 & 0.0020 & $5.08 \mathrm{e}-05$ & 29.60 & 0.999 \\
$x_{S}$ & 360.06 & 0.8658 & 0.0205 & 29.63 & 0.999 \\
$y_{S}$ & 342.44 & 0.7878 & 0.0174 & 29.18 & 0.999 \\
$x_{L}$ & 267.98 & 0.9659 & 0.0203 & 23.79 & 0.999 \\
$y_{L}$ & 304.69 & 0.8072 & 0.0178 & 27.11 & 0.999 \\
\hline
\end{tabular}

Notes.

${ }^{\mathrm{a}}$ The mean values estimated from the chain of $5 \times 10^{5}$ simulations.

b The standard deviations.

c An estimate of the Monte Carlo error.

${ }^{d}$ The integrated autocorrelation time.

e A simple test for a null hypothesis that the chain has converged.

and the gamma-ray emission is robust to the large spread in values of the Hubble constant.

Application of the method to other sources may not be as straightforward. For example, fewer constraints like well-resolved images together with time delays at the same frequency. Light curves may not be as well sampled as at gamma-rays. The radio data might be insufficient to reconstruct the projection of the jet. Furthermore, observations of relatively nearby sources show that jets can be bent, thus introducing additional uncertainty in measuring distances between emitting regions.

\subsection{Comparison with PKS1830-211}

PKS 1830-211 is the only other gravitationally lensed gamma-ray blazar known currently. Analysis of the gammaray time delays enable resolution of the origin of gamma-ray flares at the $\sim 10$ mas level, corresponding to $\sim 100 \mathrm{pc}$ in the source plane (Barnacka et al. 2015a). In this case, the spatial resolution is limited by the accuracy of time delay, $\sim 0.5$ days.

Flare 1 of B2 $0218+35$ was longer and brighter than the flares of PKS 1830-211. The excellent photon statistics of Flare 1 allow measurement of the time delay with an accuracy of $\sim 0.1$ days, an improvement by a factor of 5 relative to PKS 1830-211.

In PKS 1830-211 there is no Einstein ring. The pseudo-ringlike structure composed of images of the radio jet only allow derivation of a boundary limiting the jet projection in the source plane.

In contrast with PKS 1830-211, the well-resolved radio images and the more accurate gamma-ray time delay enable relative localization of the radio and gamma-ray sources if the Hubble constant is sufficiently well-known from some independent technique. We can separate Flare 1 from the core in B2 $0218+35$ at the $\sim 3 \sigma$ level because the Hubble parameter is measured with $\sim 2 \%$ accuracy using the cosmic microwave background fluctuations (Planck Collaboration et al. 2014).

Future measurement of the Hubble parameter with an accuracy of $\sim 1 \%$ (Bennett et al. 2014) will allow resolution of the gamma-ray emission at even greater significance, $\sim 6 \sigma$.

\subsection{The Spatial Origin of Gamma-ray Flares and Source Physics}

The spatial origin of gamma-ray flares is a subject of debate (Tavecchio et al. 2010; Nalewajko et al. 2014a). Some observations indicate that flares are produced upstream from 


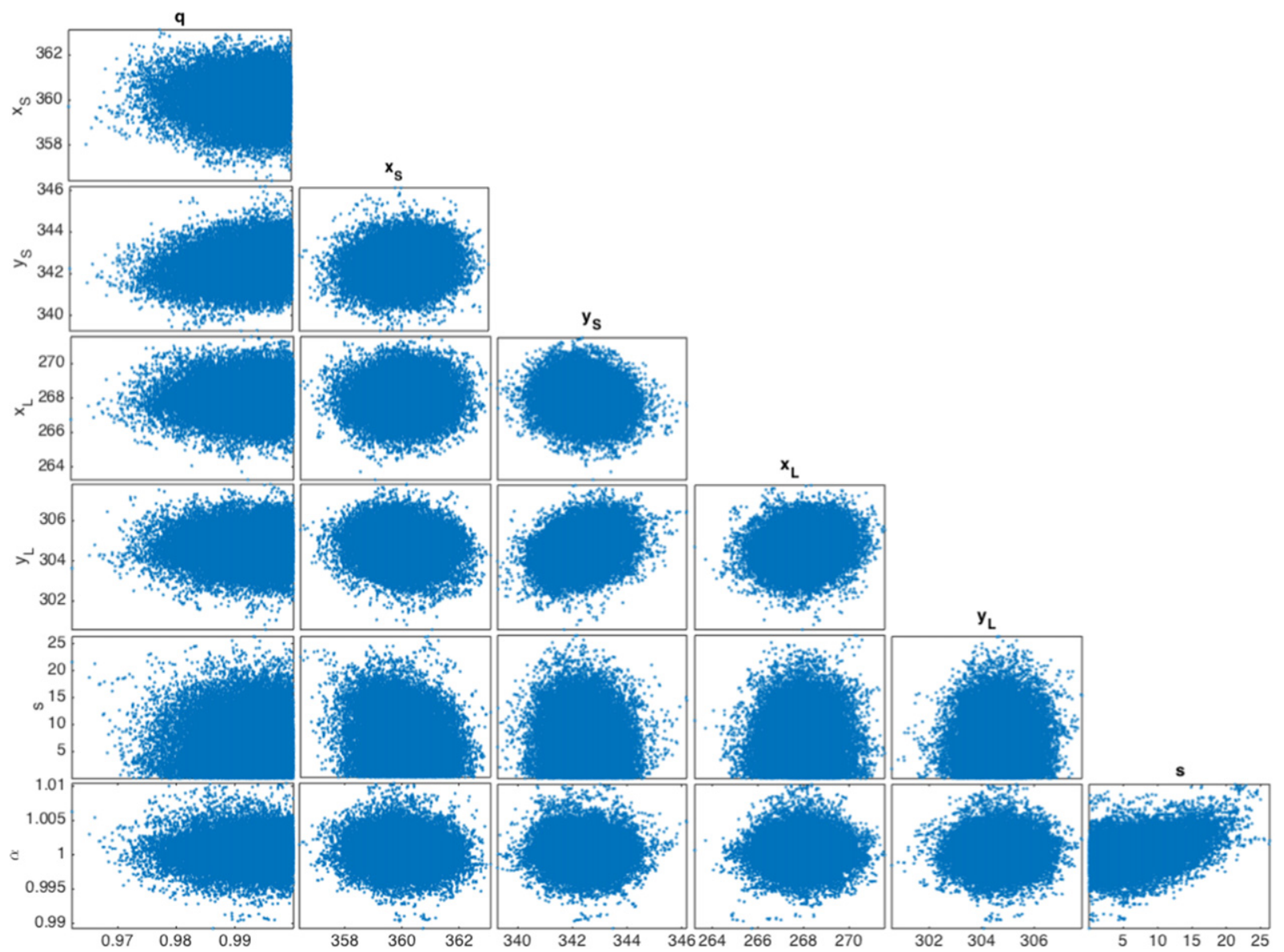

Figure 12. Pairwise scatterplots showing the bivariate marginal distributions for a softened power-law potential simulated using the Metropolis-Hastings chains.

the resolved radio core (Marscher et al. 2008, 2010; Karamanavis et al. 2015). However, most theoretical models assume that dissipation takes place within a sub-parsec scale from the central engine, where the plasma is denser and the sources of seed photons for inverse-Compton radiation are abundant (Tanaka et al. 2011; Barnacka et al. 2014b; Nalewajko et al. 2014b; Hovatta et al. 2015).

Only radio telescopes can resolve scales smaller than $100 \mathrm{pc}$. Radio telescopes probe synchrotron radiation produced in optically thick parts of the jet. The radio core is often interpreted as the location where the jet opacity to synchrotron self-absorption is 1 (Blandford \& Königl 1979). Observationally, the radio core is the region of peak intensity generated by a compact component at the apparently upstream end of the jet (Marscher 2008; Haga et al. 2015).

Measurement of the distance between a radio core and the central engine is difficult. These measurements have only been possible for nearby sources with prominent two-sided jets. Observing outflows in two opposite directions then constrains the position of the central engine (Haga et al. 2015).

For gamma-ray blazars the two-sided jet is presumably present, but the counterjet is too faint for detection because of relativistic beaming. Thus, in blazars, we observe only one sided-jets. As summarized by Haga et al. (2015), the general picture is that a central engine exists somewhere upstream of observed cores. B2 $0218+35$ provides the first direct observational constraint.

In $\mathrm{B} 20218+35$ the bright gamma-ray flare occurred upstream in the jet at the projected distance of $51.2 \pm 8.8 \mathrm{pc}$ from the $15 \mathrm{GHz}$ radio core. Thus, the central engine must be at least this far away from the radio core. The VLBA follow-up of the bright gamma-ray flare did not reveal any significant flux density variability at frequencies of 15 and $22 \mathrm{GHz}$ (Spingola et al. 2016). The Hubble tuning approach we use can enable measurement of the apparent velocities of gamma-ray emitting knots. Based on this measurement, we can predict the time of a plausible interaction with the radio core. For B2 $0218+35$ we predict increased emission at gamma-ray and/or radio wavelengths within a year of 2016 July.

\subsection{Gravitational Lensing and X-Ray Sources}

The improvement of angular resolution at X-ray wavelengths with Chandra enabled the discovery of high-energy extragalactic jets extending over hundreds of kiloparsecs (Chartas et al. 2000; Schwartz et al. 2000; Sambruna et al. 2002, 2004, 2006; Siemiginowska et al. 2002; Marshall et al. 2005; Harris \& Krawczynski 2006; Tavecchio et al. 2007). In fact, our investigations of the structure of lensed gamma-ray sources were inspired by Chandra's discovery of flaring emission from $H S T$-1, a knot of strong X-ray emission displaced from the core in the nearby galaxy M87 (Harris et al. 2006). Recently, deep Chandra observations of Pictor A revealed the high-energy flares located in knots displaced along the jet (Hardcastle et al. 2015).

For sources at redshift $\sim 1$, the Chandra resolution of 0.5 arcsec corresponds to $4 \mathrm{kpc}$. Thus, knots like $H S T$ - 1 cannot be resolved. Improvement in angular resolution of at least two orders of magnitude is needed to resolve these sources and to explore their evolution. 

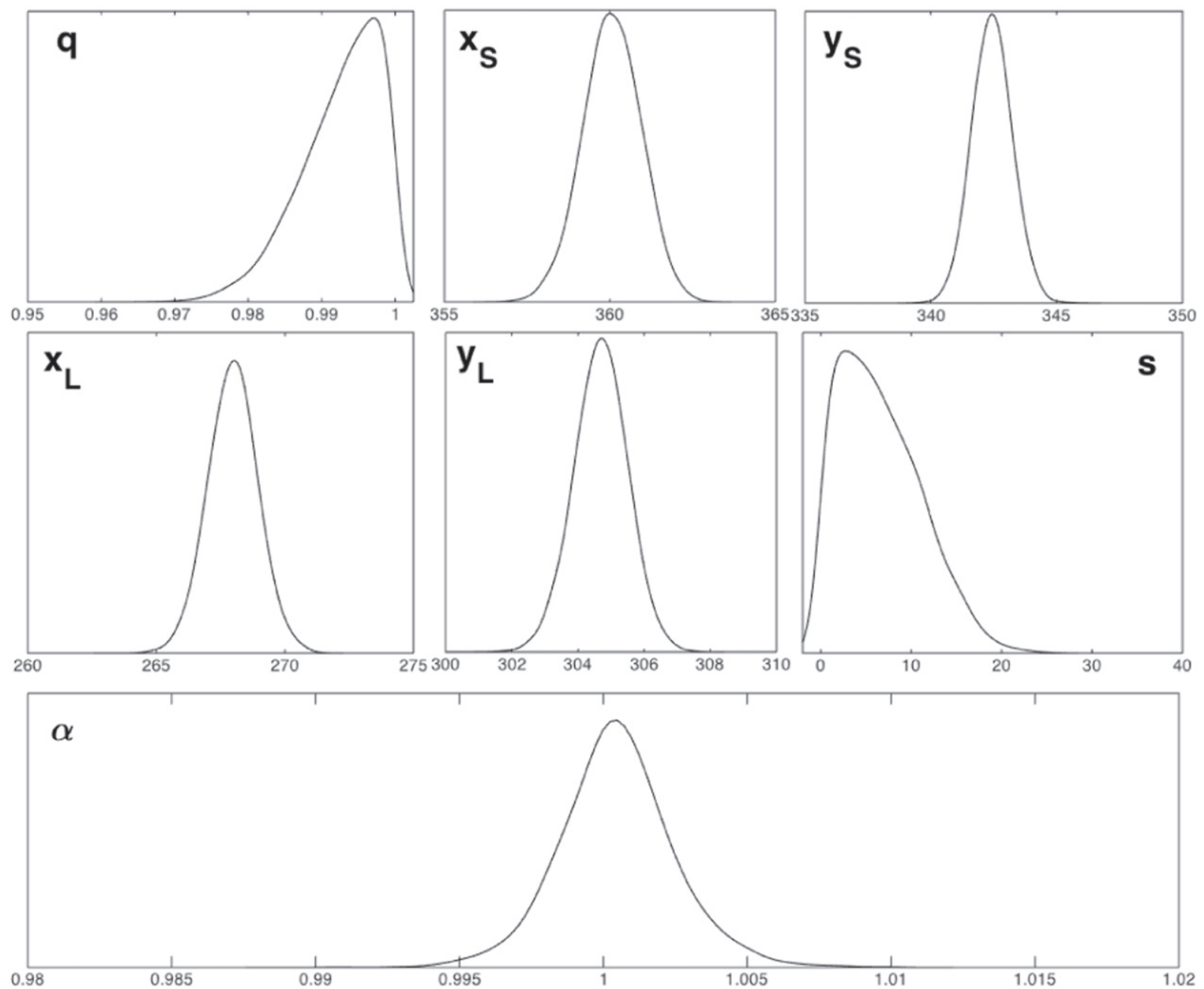

Figure 13. The univariate marginalized probabilities for all parameters of lens parameters of the softened power-law potential.

There are, however, 20 gravitationally lensed quasars with associated X-ray emission. The sources have been monitored in searches for microlensing (Chartas et al. 2002, 2012; Dai et al. 2003; Chen et al. 2012; Mosquera et al. 2013). These data and future monitoring of variable X-ray sources may enable measurement of time delays.

These sources have been also monitored at radio and optical wavelengths. Combining these observations along the lines we have followed for B2 $0218+35$ may enable reconstruction of these sources based on lensing models. If displacements of the $\mathrm{X}$-ray emitting regions along the jets are common, they pose challenges to understanding of the particle acceleration mechanism. They may also increase our understanding of the distribution of Hubble constants derived from time delays (Barnacka et al. 2015b).

\subsection{Gravitational Lensing and SKA}

SKA will observe thousands of gravitationally lensed quasars with a resolution of $\sim 2$ mas at $10 \mathrm{GHz}$, and $\sim 20$ mas at $1 \mathrm{GHz}$ (Dewdney et al. 2009; Godfrey et al. 2012; McKean et al. 2015, p. 84). These radio observations will provide a foundation for reconstructing the mass distribution of lenses and the positions of radio cores.

All radio quasars have X-ray emission (Risaliti \& Lusso 2015). Thus, among these lensed systems, there will be a large population of quasars with variable high-energy emission where time delays can be measured.

An ensemble of high-energy quasars with measured time delays and reconstructed source structure based on robust lensing models will enable investigation of the origin of $\mathrm{X}$-ray radiation and the connection between the radio and high-energy emission. The large redshift range of lensed quasars should provide constraints on the co-evolution of radio and high-energy jets.

\section{CONCLUSIONS}

B2 $0218+35$ is one of only two known gravitationally lensed systems detected at gamma-rays. We reconstruct the mass distribution of its lensing galaxy and the properties of the jet based on well-resolved radio observations. We use the dense Fermi-LAT light curves to measure time delays for two gamma-ray flares. The position of the mirage images, the time delay, and the independently known Hubble constant enable measurement of the spatial offset between the gamma-ray emission region and the radio core.

Our reconstruction of the lensed source shows that the extended flare (Flare 1) is displaced from the radio core at the $\sim 3 \sigma$ level. The displacement is upstream from the jet providing the first direct observational constraint on the location of the central engine relative to the radio core for blazars. 
Table 6

The Expected Differences in Time Delay and Magnification Ratios for the Monte Carlo Simulations

\begin{tabular}{|c|c|c|c|c|c|c|c|}
\hline $\begin{array}{l}\text { Parameter within } 1 \sigma \\
\text { SIS }\end{array}$ & $\begin{array}{c}d \Delta t / d r \\
0.61 \text { days } / 6 \text { mas }\end{array}$ & $\begin{array}{c}\text { dRatio } / d r \\
0.55 / 6 \text { mas }\end{array}$ & $\begin{array}{c}\text { Dist (mas) } \\
1.25 \\
\end{array}$ & $\begin{array}{l}\text { Parameter within } 3 \sigma \\
\text { SIS }\end{array}$ & $\begin{array}{c}d \Delta t / d r \\
0.61 \text { days } / 6 \text { mas }\end{array}$ & $\begin{array}{c}d \text { Ratio } / d r \\
0.55 / 6 \text { mas }\end{array}$ & Dist (mas) \\
\hline$\alpha=0.998$ & 0.613 days $/ 6$ mas & $0.47 / 6$ mas & 4.5 & $\alpha=0.994$ & 0.62 days $/ 6$ mas & $0.48 / 6$ mas & 12 \\
\hline$\alpha=1.002$ & 0.61 days $/ 6$ mas & $0.5 / 6$ mas & 4.6 & $\alpha=1.006$ & 0.6 days $/ 6$ mas & $0.48 / 6$ mas & 12 \\
\hline$s=4.5$ & 0.61 days $/ 6$ mas & $0.55 / 6$ mas & 1.8 & $s=13.5$ & 0.61 days $/ 6$ mas & $0.55 / 6$ mas & 1.8 \\
\hline$q=1.0055$ & 0.58 days $/ 6$ mas & $0.5 / 6$ mas & 2.6 & $q=1.0165$ & 0.7 days $/ 6$ mas & $0.43 / 6$ mas & 4.6 \\
\hline
\end{tabular}

A shorter flare (Flare 2) may be an event following Flare 1. If Flare 1 and Flare 2 are indeed connected then the knot which produced these gamma-ray flares moves at apparent velocity of $\sim 70 \mathrm{c}$. Thus the model makes a testable prediction. There should be an interaction with the radio core within a year of 2016 July.

The only other known gravitationally lensed gamma-ray blazar is PKS 1830-211. This source also has complex structure with gamma-ray flares originating from multiple regions along the jet (Barnacka et al. 2011, 2015a).

Lensed high-energy sources monitored with detectors like Chandra, Swift and NuSTAR offer rich opportunities to extend this powerful lens modeling approach to other sources. There are more that 20 known lensed quasars with associated X-ray emission. Some of these systems already have enough observations to reconstruct the mass distribution of the lens. Further monitoring will enable measurement of time delays with X-ray detectors.

In the near future, SKA will resolve thousands of radio images of gravitationally lensed quasars. Many of these quasars will also have prominent variable X-ray emission, allowing recovery of time delays. This ensemble of observations combined with the power of strong gravitational lensing will probe the origin of $\mathrm{X}$-ray radiation, its connection to radio emission, and the cosmic evolution of jets at radio and high energies.

We thank Scott Kenyon and Dan Schwartz for valuable comments. We thank the referee for providing valuable comments.

$\mathrm{AB}$ is supported by NASA through Einstein Postdoctoral Fellowship. MJG is supported by the Smithsonian Institution.

\section{APPENDIX \\ MONTE CARLO SIMULATIONS AND LENS MODELING}

Selecting the class of models is a key part of lens modeling. A simple and useful mass model of a galaxy acting as a lens is an isothermal model with density $\rho \propto r^{-2}$ and a flat rotation curve. Moreover, the isothermal profile is consistent with a large range of observations of spiral and elliptical galaxies (Fabbiano 1989; Maoz \& Rix 1993; Kochanek 1995; Rix et al. 1997; Cohn et al. 2001; Treu \& Koopmans 2002; Koopmans \& Treu 2003).

Here, our goal is to find a unique mass distribution for the lens. Therefore, we investigate a lens profile allowing for a more complex mass distribution. We use a softened power-law potential (Keeton 2001, Equation (25)):

$$
\phi=b\left(s^{2}+x^{2}+y^{2} / q^{2}\right)^{\alpha / 2}-b s^{\alpha},
$$

where $b$ is the Einstein radius, $s$ is a scale radius of a flat core (in mas), $q$ is the projected axis ratio, and $\alpha$ is a power-law exponent. The softened power-law potential with $s=0, q=1$, and $\alpha=1$ reduces to the SIS potential.

Table 4 shows the range of parameters we explore using Monte Carlo simulations. These range of parameters are constrained based on the set of radio and optical observations described in Section 3.1.
We use Monte Carlo simulations to explore lens models defined by Equation (8) varying in the range of parameters listed in Table 4 . We use the MCMC ${ }^{8}$ toolbox within MATLAB. This toolbox generates and analyzes Metropolis-Hastings MCMC chains using a multivariate Gaussian proposal distribution. Table 5 and Figures 12 and 13 shows the results of the MC simulations. The derived parameters are very close to the ideal SIS where $s=0, q=1$, and $\alpha=1$.

The Monte Carlo simulations show that the best global solution for the lens model for B2 $0218+35$ is the SIS. To exclude other solutions, we have run an additional $10^{6} \mathrm{MC}$ simulation uniformly sampled over the range of parameters from Table 4 . We identified local minima with parameters for the lens potential that reproduce the position of the images. All models that reconstruct the image within at least 5 mas are very close the SIS. Moreover, all models with a more complex gravitational potential that provide a reasonable reconstruction of the mirage images fail to reproduce the radio time delay.

Precise reconstruction of the distance between the emitting regions of the well resolved mirage images, and the emitting region with the well measured time delay rely on knowledge of the mass distribution of the lens. Different lens models result in different rates of change of the time delay as a function of the position of the source. In principle, this rate is the source of systematics in calibrating the distance between the emitting regions.

We calculate the rates of change in the time delay around the source position for different parameters of the lens model. We calculate the change in the time delay for source positions moved by 6 mas in the radial direction. We calculate the expected change in the time delay for models deviating from the SIS. We use the error in the lens parameters estimated from the Monte Carlo simulations (Table 5). We investigate lens models with parameters $\alpha, s$, and $q$ within $1 \sigma$ and $3 \sigma$ from the SIS.

Table 6 shows the expected differences in time delays and magnification ratios. We also list the resulting difference between the reconstructed position of the images and positions of the observed images. For example, even a change as small as 0.002 in the $\alpha$ parameter results in a significant change in the reconstructed image positions. The calculations summarized in Table 6 demonstrate that the estimated offset between the radio core and region of the gamma-ray flare of $6.6 \pm 1.1$ mas does not suffer from systematics in the lens modeling. The offset is estimated with $\sim 20 \%$ accuracy. As demonstrated in Table 6, the lens model cannot introduce uncertainties of more that $5 \%$. Therefore, our estimation of the offset between the radio core and gamma-ray flare is robust.

\section{REFERENCES}

Atwood, W. B., Abdo, A. A., Ackermann, M., et al. 2009, ApJ, 697, 1071

\footnotetext{
8 http://helios.fmi.fi/ lainema/mcmc/
} 
Barnacka, A. 2013, arXiv:1307.4050

Barnacka, A., Geller, M. J., Dell'Antonio, I. P., \& Benbow, W. 2014a, ApJ, 788, 139

Barnacka, A., Geller, M. J., Dell'Antonio, I. P., \& Benbow, W. 2015a, ApJ, 809,100

Barnacka, A., Geller, M. J., Dell'Antonio, I. P., \& Benbow, W. 2015b, ApJ, 799, 48

Barnacka, A., Glicenstein, J.-F., \& Moudden, Y. 2011, A\&A, 528, L3

Barnacka, A., Moderski, R., Behera, B., Brun, P., \& Wagner, S. 2014b, A\&A, 567, A113

Bennett, C. L., Larson, D., Weiland, J. L., \& Hinshaw, G. 2014, ApJ, 794, 135

Biggs, A. D., Browne, I. W. A., Helbig, P., et al. 1999, MNRAS, 304, 349

Biggs, A. D., Browne, I. W. A., Jackson, N. J., et al. 2004, MNRAS, 350, 949

Biggs, A. D., Browne, I. W. A., Muxlow, T. W. B., \& Wilkinson, P. N. 2001, MNRAS, 322, 821

Biggs, A. D., Wucknitz, O., Porcas, R. W., et al. 2003, MNRAS, 338, 599

Blandford, R., \& Narayan, R. 1986, ApJ, 310, 568

Blandford, R. D., \& Königl, A. 1979, ApJ, 232, 34

Bogert, B. P., Healy, M. J. R., \& Tukey, J. W. 1963, in Proc. Symp. on Time Series Analysis, The Quefrency Analysis of Time Series for Echoes: Cepstrum, Pseudo Autovariance, Cross-Cepstrum and Saphe Cracking, ed. M. Rosenblat (New York: Wiley), 209

Broderick, A. E., \& Tchekhovskoy, A. 2015, ApJ, 809, 97

Browne, I. W. A., Patnaik, A. R., Walsh, D., \& Wilkinson, P. N. 1993, MNRAS, 263, L32

Cerutti, B., Werner, G. R., Uzdensky, D. A., \& Begelman, M. C. 2012, ApJL, 754, L33

Chartas, G., Agol, E., Eracleous, M., et al. 2002, ApJ, 568, 509

Chartas, G., Kochanek, C. S., Dai, X., et al. 2012, ApJ, 757, 137

Chartas, G., Worrall, D. M., Birkinshaw, M., et al. 2000, ApJ, 542, 655

Chávez, R., Terlevich, E., Terlevich, R., et al. 2012, MNRAS, 425, L56

Chen, B., Dai, X., Kochanek, C. S., et al. 2012, ApJ, 755, 24

Cheung, C. C., Larsson, S., Scargle, J. D., et al. 2014, ApJL, 782, L14

Cohen, A. S., Hewitt, J. N., Moore, C. B., \& Haarsma, D. B. 2000, ApJ, 545,578

Cohen, J. G., Lawrence, C. R., \& Blandford, R. D. 2003, ApJ, 583, 67

Cohn, J. D., Kochanek, C. S., McLeod, B. A., \& Keeton, C. R. 2001, ApJ, 554, 1216

Corbett, E. A., Browne, I. W. A., Wilkinson, P. N., \& Patnaik, A. 1996, in IAU Symp. 173, Astrophysical Applications of Gravitational Lensing, ed. C. S. Kochanek, \& J. N. Hewitt (Dordrecht: Kluwer), 37

Dai, X., Chartas, G., Agol, E., Bautz, M. W., \& Garmire, G. P. 2003, ApJ, 589, 100

Dewdney, P. E., Hall, P. J., Schilizzi, R. T., \& Lazio, T. J. L. W. 2009, in IEEE Proc. 97, 1482

Efstathiou, G. 2014, MNRAS, 440, 1138

Fabbiano, G. 1989, ARA\&A, 27, 87

Freedman, W. L., \& Madore, B. F. 2010, ARA\&A, 48, 673

Freedman, W. L., Madore, B. F., Gibson, B. K., et al. 2001, ApJ, 553, 47

Freedman, W. L., Madore, B. F., Scowcroft, V., et al. 2012, ApJ, 758, 24

Giannios, D., Uzdensky, D. A., \& Begelman, M. C. 2009, MNRAS, 395, L29

Godfrey, L. E. H., Bignall, H., Tingay, S., et al. 2012, PASA, 29, 42

Guo, F., Li, H., Daughton, W., \& Liu, Y.-H. 2014, PhRvL, 113, 155005

Guo, F., Liu, Y.-H., Daughton, W., \& Li, H. 2015, ApJ, 806, 167

Haario, H., Laine, M., Mira, A., \& Saksman, E. 2006, Statistics and Computing, 16, 339

Haga, T., Doi, A., Murata, Y., et al. 2015, ApJ, 807, 15

Hardcastle, M. J., Lenc, E., Birkinshaw, M., et al. 2015, arXiv:1510.08392

Harris, D. E., Cheung, C. C., Biretta, J. A., et al. 2006, ApJ, 640, 211

Harris, D. E., \& Krawczynski, H. 2006, ARA\&A, 44, 463

Hoshino, M. 2012, PhRvL, 108, 135003

Hovatta, T., Petropoulou, M., Richards, J. L., et al. 2015, MNRAS, 448, 3121

Jackson, N., Xanthopoulos, E., \& Browne, I. W. A. 2000, MNRAS, 311, 389

Jaroschek, C. H., Treumann, R. A., Lesch, H., \& Scholer, M. 2004, PhPl, 11,1151

Jorstad, S. G., Marscher, A. P., Lister, M. L., et al. 2005, AJ, 130, 1418

Karamanavis, V., Fuhrmann, L., Krichbaum, T. P., et al. 2015, arXiv: 1511.01085

Keeton, C. R. 2001, arXiv:astro-ph/0102341

Kirk, J. G., \& Skjæraasen, O. 2003, ApJ, 591, 366

Kneib, J. P. 2014, Manual of the LensTool Program, https://projets.lam.fr/ projects/lenstool/wiki

Kochanek, C. S. 1995, ApJ, 445, 559

Kochanek, C. S., \& Dalal, N. 2004, ApJ, 610, 69

Komissarov, S. S., Barkov, M. V., Vlahakis, N., \& Königl, A. 2007, MNRAS, 380,51
Koopmans, L. V. E., \& Treu, T. 2003, ApJ, 583, 606

Larchenkova, T. I., Lutovinov, A. A., \& Lyskova, N. S. 2011, AstL, 37, 233

Lehár, J., Falco, E. E., Kochanek, C. S., et al. 2000, ApJ, 536, 584

Lister, M. L., Aller, M. F., Aller, H. D., et al. 2013, AJ, 146, 120

Lister, M. L., Aller, M. F., Aller, H. D., et al. 2015, ApJL, 810, L9

Lyubarsky, Y. E. 2005, MNRAS, 358, 113

Lyutikov, M., \& Uzdensky, D. 2003, ApJ, 589, 893

MacDonald, N. R., Marscher, A. P., Jorstad, S. G., \& Joshi, M. 2015, ApJ, 804, 111

Mao, S., \& Schneider, P. 1998, MNRAS, 295, 587

Maoz, D., \& Rix, H.-W. 1993, ApJ, 416, 425

Marscher, A. P. 2008, in ASP Conf. Ser. 386, Extragalactic Jets: Theory and Observation from Radio to Gamma Ray, ed. T. A. Rector, \& D. S. De Young (San Francisco, CA: ASP), 437

Marscher, A. P., Jorstad, S. G., D’Arcangelo, F. D., et al. 2008, Natur, 452, 966

Marscher, A. P., Jorstad, S. G., Larionov, V. M., et al. 2010, ApJL, 710, L126

Marshall, H. L., Schwartz, D. A., Lovell, J. E. J., et al. 2005, ApJS, 156, 13

Mattox, J. R., Bertsch, D. L., Chiang, J., et al. 1996, ApJ, 461, 396

McKean, J., Jackson, N., Vegetti, S., et al. 2015, Advancing Astrophysics with the Square Kilometre Array (Giardini Naxos, Italy: AASKA14), 84, http:// pos.sissa.it/cgi-bin/reader/conf.cgi?confid $=215$

McKinney, J. C. 2006, MNRAS, 368, 1561

Metcalf, R. B., \& Amara, A. 2012, MNRAS, 419, 3414

Mittal, R., Porcas, R., \& Wucknitz, O. 2007, A\&A, 465, 405

Moscibrodzka, M., Falcke, H., \& Shiokawa, H. 2015, arXiv:1510.07243

Mosquera, A. M., Kochanek, C. S., Chen, B., et al. 2013, ApJ, 769, 53

Nalewajko, K., Begelman, M. C., \& Sikora, M. 2014a, ApJ, 789, 161

Nalewajko, K., Giannios, D., Begelman, M. C., Uzdensky, D. A., \& Sikora, M. 2011, MNRAS, 413, 333

Nalewajko, K., Sikora, M., \& Begelman, M. C. 2014b, ApJL, 796, L5

Nalewajko, K., Uzdensky, D. A., Cerutti, B., Werner, G. R., \& Begelman, M. C. 2015, arXiv:1508.02392

Narayan, R., \& Bartelmann, M. 1996, arXiv:astro-ph/9606001

Nolan, P. L., Abdo, A. A., Ackermann, M., et al. 2012, ApJS, 199, 31

O'Dea, C. P., Baum, S. A., Stanghellini, C., et al. 1992, AJ, 104, 1320

Patnaik, A. R., Browne, I. W. A., King, L. J., et al. 1993, MNRAS, 261, 435

Patnaik, A. R., Browne, I. W. A., Wilkinson, P. N., \& Wrobel, J. M. 1992, MNRAS, 254, 655

Patnaik, A. R., Porcas, R. W., \& Browne, I. W. A. 1995, MNRAS, 274, L5

Peng, Z. Y., Yin, Y., Bi, X. W., et al. 2010, ApJ, 718, 894

Planck Collaboration, Ade, P. A. R., Aghanim, N., et al. 2014, A\&A, 571, A16

Proga, D. 2005, ApJ, 629, 397

Riess, A. G., Macri, L., Casertano, S., et al. 2011a, ApJ, 730, 119

Riess, A. G., Macri, L., Casertano, S., et al. 2011b, ApJ, 732, 129

Risaliti, G., \& Lusso, E. 2015, Exploring the Hot and Energetic Universe, ed. M. Ehle, 26

Rix, H.-W., de Zeeuw, P. T., Cretton, N., van der Marel, R. P., \& Carollo, C. M. 1997, ApJ, 488, 702

Saito, S., Stawarz, Ł., Tanaka, Y. T., et al. 2013, ApJL, 766, L11

Sambruna, R. M., Gambill, J. K., Maraschi, L., et al. 2004, ApJ, 608, 698

Sambruna, R. M., Gliozzi, M., Donato, D., et al. 2006, ApJ, 641, 717

Sambruna, R. M., Maraschi, L., Tavecchio, F., et al. 2002, ApJ, 571, 206

Schwartz, D. A., Marshall, H. L., Lovell, J. E. J., et al. 2000, ApJL, 540, L69

Siemiginowska, A., Bechtold, J., Aldcroft, T. L., et al. 2002, ApJ, 570, 543

Sironi, L., \& Spitkovsky, A. 2014, ApJL, 783, L21

Spingola, C., Dallacasa, D., Orienti, D., et al. 2016, MNRAS, 457, 2263

Stawarz, Ł., \& Ostrowski, M. 2002, ApJ, 578, 763

Suyu, S. H., Auger, M. W., Hilbert, S., et al. 2013, ApJ, 766, 70

Suyu, S. H., Marshall, P. J., Auger, M. W., et al. 2010, ApJ, 711, 201

Tanaka, Y. T., Stawarz, Ł., Thompson, D. J., et al. 2011, ApJ, 733, 19

Tavecchio, F., Ghisellini, G., Bonnoli, G., \& Ghirlanda, G. 2010, MNRAS, 405, L94

Tavecchio, F., Maraschi, L., Wolter, A., et al. 2007, ApJ, 662, 900

Tchekhovskoy, A., \& Bromberg, O. 2015, arXiv:1512.04526

Tchekhovskoy, A., Narayan, R., \& McKinney, J. C. 2010, ApJ, 711, 50

Tchekhovskoy, A., Narayan, R., \& McKinney, J. C. 2011, MNRAS, 418, L79

Treu, T., \& Koopmans, L. V. E. 2002, ApJ, 575, 87

Vovk, I., \& Neronov, A. 2015, arXiv:1507.01092

Wucknitz, O., Biggs, A. D., \& Browne, I. W. A. 2004, MNRAS, 349, 14

York, T., Jackson, N., Browne, I. W. A., Wucknitz, O., \& Skelton, J. E. 2005, MNRAS, 357, 124

Zenitani, S., \& Hoshino, M. 2001, ApJL, 562, L63

Zenitani, S., \& Hoshino, M. 2007, ApJ, 670, 702

Zitrin, A., Broadhurst, T., Umetsu, K., et al. 2009, MNRAS, 396, 1985

Zitrin, A., Meneghetti, M., Umetsu, K., et al. 2013, ApJL, 762, L30

Zweibel, E. G., \& Yamada, M. 2009, ARA\&A, 47, 291 